\title{
Non-semi-regular quantum groups coming from number theory
}

\author{
Communications in Mathematical Physics 235 (1) (2003), 139-167. \\ by Saad Baaj, Georges Skandalis and Stefaan Vaes

\begin{abstract}
Laboratoire de Mathématiques Pures; Université Blaise Pascal; Bâtiment de Mathématiques; F-63177 Aubière Cedex (France) e-mail: Saad.Baaj@math.univ-bpclermont.fr

Institut de Mathématiques de Jussieu; Algèbres d'Opérateurs et Représentations; 175, rue du Chevaleret; F-75013 Paris (France) e-mails: skandal@math.jussieu.fr and vaes@math.jussieu.fr
\end{abstract}

\begin{abstract}
In this paper, we study $\mathrm{C}^{*}$-algebraic quantum groups obtained through the bicrossed product construction. Examples using groups of adeles are given and they provide the first examples of locally compact quantum groups which are not semi-regular: the crossed product of the quantum group acting on itself by translations does not contain any compact operator. We describe all corepresentations of these quantum groups and the associated universal $\mathrm{C}^{*}$-algebras. On the way, we provide several remarks on $\mathrm{C}^{*}$-algebraic properties of quantum groups and their actions.
\end{abstract}

\section{Introduction}

What is the quantum analogue of a locally compact group? Several authors addressed this question and came out with various answers. G.I. Kac, Kac-Vainerman and Enock-Schwartz gave a quite satisfactory set of axioms in $[7,9,11]$ defining what Enock-Schwartz called Kac algebras. In the 1980's, new examples, which were certainly to be considered as quantum groups, but were not Kac algebras, were constructed, see e.g. [22]. Many efforts were then made to enlarge the definition of Kac algebras.

Already in the Kac algebra setting, it was known that all the information on the quantum group could be encoded in one object: this was called a multiplicative unitary in [2]. It was then quite natural to try and make constructions with a multiplicative unitary as a starting point.

In order to be able to construct $\mathrm{C}^{*}$-algebras out of a multiplicative unitary, some additional assumptions were made: this multiplicative unitary was assumed to be regular in [2], semi-regular in [1], manageable in $[21], \ldots$ In fact, a multiplicative unitary can really be quite singular: in Remark 4.5 below, we give an example of a multiplicative unitary which should certainly not be considered as the quantum analogue of a locally compact group.

In $[14,15]$, a definition of a locally compact quantum group along the lines of Kac algebras, but with a weaker set of axioms, was given and was shown to lead to a manageable multiplicative unitary. We believe that the definitions of $[14,15]$ can be considered as the final ones, at least from the measure theoretic (von Neumann) point of view.

On the other hand, regularity and semi-regularity are natural conditions and present some additional features - that will be discussed below. In particular, regularity is very much connected with the Takesaki-Takai duality.

All this leaves us with many questions: what are the relations between the properties of semi-regularity and manageability? We actually really tried hard to prove that these properties are equivalent. All previously known examples were locally compact quantum groups whose multiplicative unitary was semi-regular.

The main result of this paper is the construction of a locally compact quantum group whose multiplicative unitary is not semi-regular. This is done using a construction which goes back to G.I. Kac [10] and was used by several authors $[2,3,16,18,19,20, \ldots]$. Let $G$ be a locally compact group and let $G_{1}$ and $G_{2}$ be closed subgroups such that the map $\theta:\left(x_{1}, x_{2}\right) \mapsto x_{1} x_{2}$ from $G_{1} \times G_{2}$ into $G$ is a measure class isomorphism. Associated to this situation, there is a locally compact quantum group. We will show that its associated 
multiplicative unitary is semi-regular if and only if $\theta$ is a homeomorphism from $G_{1} \times G_{2}$ onto an open subset of $G$. Moreover, we will be able to identify all the associated operator algebras.

In fact, in the examples considered up to now, $G$ was a real Lie group and one could easily see that the associated multiplicative unitary is always semi-regular (by differentiability considerations).

The examples that we consider here are just adelic analogues of these Lie groups. In particular, we will consider the case were $G$ is an adelic $a x+b$ group and $G_{1}, G_{2}$ are natural subgroups.

Note that in the non-regular case, the image of $\theta$ is a countable union of compact sets with empty interior and the complement of the image of $\theta$ has measure 0 . Although this is a relatively common phenomenon in topology, it was quite a surprise to us to encounter it in the locally compact group case. It was taken for granted in [3] that such a phenomenon could not occur. Because of this, the main result in [3] is incorrect as stated. One has either to add the extra assumption that the associated multiplicative unitary is semi-regular, or to change the conclusion: the product map $\left(g_{1}, g_{2}\right) \mapsto g_{1} g_{2}$ is not a homeomorphism from $G_{1} \times G_{2}$ onto a dense open subset of $G$ but a measure class isomorphism. This is discussed in Subsection a).

We conclude the introduction by explaining the structure of the paper. In Section 2, we recall the definition and main properties of multiplicative unitaries and locally compact quantum groups. In Section 3 , we introduce the most general definition of a matched pair of locally compact groups. We describe the associated locally compact quantum groups obtained through the bicrossed product construction. We compute the different associated $\mathrm{C}^{*}$-algebras, as well as the corepresentations and the covariant representations. We give necessary and sufficient conditions for (semi-)regularity. In Section 5, we give several examples, providing the first examples of locally compact quantum groups that are not semi-regular. Finally, in Section 5, we give different characterizations of (semi-)regularity and we consider some $\mathrm{C}^{*}$-algebraic properties of coactions.

\section{Preliminaries}

In this paper, all locally compact groups are supposed to be second countable.

When $X$ is a subset of a Banach space, we denote by $[X]$ the norm closed linear span of $X$. We denote by $\mathcal{K}(H)$ and $\mathcal{L}(H)$ the compact, resp. the bounded operators on a Hilbert space $H$. We use $\Sigma$ to denote the flip map from $H \otimes K$ to $K \otimes H$, when $H$ and $K$ are Hilbert spaces.

By $\otimes$, we denote several types of tensor products: minimal tensor products of $\mathrm{C}^{*}$-algebras, von Neumann algebraic tensor products or Hilbert space tensor products. There should be no confusion.

\section{a) Multiplicative unitaries and locally compact quantum groups}

Multiplicative unitaries were studied in [2]. We have the following definition.

Definition 2.1. A unitary $W \in \mathcal{L}(H \otimes H)$ is called a multiplicative unitary if $W$ satisfies the pentagonal equation

$$
W_{12} W_{13} W_{23}=W_{23} W_{12}
$$

We associate to any multiplicative unitary two natural algebras $S$ and $\hat{S}$ defined by

$$
S=\left[(\omega \otimes \iota)(W) \mid \omega \in \mathcal{L}(H)_{*}\right], \quad \hat{S}=\left[(\iota \otimes \omega)(W) \mid \omega \in \mathcal{L}(H)_{*}\right] .
$$

In general, the norm closed algebras $S$ and $\hat{S}$ need not be $\mathrm{C}^{*}$-algebras, but they are when the multiplicative unitary is regular or semi-regular, see Definition 2.5 and the papers [1, 2], or when it is manageable, see [21]. In these cases, we can define comultiplications $\delta$ and $\hat{\delta}$ on the $\mathrm{C}^{*}$-algebras $S$ and $\hat{S}$ by the formulas

$$
\delta: S \rightarrow \mathrm{M}(S \otimes S): \delta(x)=W(x \otimes 1) W^{*}, \quad \hat{\delta}: \hat{S} \rightarrow \mathrm{M}(\hat{S} \otimes \hat{S}): \hat{\delta}(y)=W^{*}(1 \otimes y) W .
$$

Multiplicative unitaries appear most naturally as the right (or left) regular representation of a locally compact (l.c.) quantum group. The theory of locally compact quantum groups is developed in $[14,15]$. 
Definition 2.2. A pair $(M, \delta)$ is called a (von Neumann algebraic) l.c. quantum group when

- $M$ is a von Neumann algebra and $\delta: M \rightarrow M \otimes M$ is a normal and unital $*$-homomorphism satisfying the coassociativity relation : $(\delta \otimes \iota) \delta=(\iota \otimes \delta) \delta$.

- There exist normal semi-finite faithful weights $\varphi$ and $\psi$ on $M$ such that

$-\varphi$ is left invariant in the sense that $\varphi((\omega \otimes \iota) \delta(x))=\varphi(x) \omega(1)$ for all $x \in M^{+}$with $\varphi(x)<\infty$ and all $\omega \in M_{*}^{+}$,

- $\psi$ is right invariant in the sense that $\psi((\iota \otimes \omega) \delta(x))=\psi(x) \omega(1)$ for all $x \in M^{+}$with $\psi(x)<\infty$ and all $\omega \in M_{*}^{+}$.

There is an equivalent $\mathrm{C}^{*}$-algebraic approach to l.c. quantum groups and the link is provided by the right (or left) regular representation. So, suppose that $(M, \delta)$ is a l.c. quantum group with right invariant weight $\psi$. Represent $M$ on the GNS-space $H$ of $\psi$ and consider the subspace $\mathcal{N}_{\psi} \subset M$ of square integrable elements:

$$
\mathcal{N}_{\psi}=\left\{x \in M \mid \psi\left(x^{*} x\right)<\infty\right\} .
$$

Denote by $\Gamma: \mathcal{N}_{\psi} \rightarrow H$ the GNS-map. Then, we define a unitary $V \in \mathcal{L}(H \otimes H)$ by the formula

$$
V(\Gamma(x) \otimes \Gamma(y))=(\Gamma \otimes \Gamma)(\delta(x)(1 \otimes y)) \quad \text { for all } \quad x, y \in \mathcal{N}_{\psi} .
$$

The unitary $V$ is a multiplicative unitary and it is called the right regular representation of $(M, \delta)$. The comultiplication $\delta$ is implemented by $V$ as above: $\delta(x)=V(x \otimes 1) V^{*}$.

Although $V$ need not be regular or semi-regular in general (see the discussion below), it is always manageable (see [14]) and we have $\mathrm{C}^{*}$-algebras $S$ and $\hat{S}$ defined by Equation (2.1) and we have the comultiplications $\delta$ and $\hat{\delta}$ on the $\mathrm{C}^{*}$-algebraic level.

To compare notations between this paper and the papers $[14,15]$, we observe that in $[14,15]$, the left regular representation is the main object. The $(S, \delta)$ agrees with the $(A, \Delta)$ of [14], but $\hat{S}$ agrees with $\hat{J} \hat{A} \hat{J}$ and $\hat{\delta}(y)$ agrees with $(\hat{J} \otimes \hat{J}) \hat{\Delta}(\hat{J} y \hat{J})(\hat{J} \otimes \hat{J})$.

\section{b) Representations and corepresentations}

Next, we recall the notion of a representation and a corepresentation of a multiplicative unitary, see Definition A.1 in [2].

Definition 2.3. Suppose that $W$ is a multiplicative unitary on the Hilbert space $H$. We call $x \in \mathcal{L}(K \otimes H)$ a representation of $W$ on the Hilbert space $K$ if $x_{12} \quad x_{13} \quad W_{23}=W_{23} x_{12}$. We call $y \in \mathcal{L}(H \otimes K)$ a corepresentation on the Hilbert space $K$ if $W_{12} y_{13} y_{23}=y_{23} W_{12}$.

We remark that, if $W$ is the (left or right) regular representation of a l.c. quantum group, then a representation $x$ satisfies $x \in \mathrm{M}(\mathcal{K}(K) \otimes S)$ and a corepresentation $y$ satisfies $y \in \mathrm{M}(\hat{S} \otimes \mathcal{K}(K))$. The defining relations become $(\iota \otimes \delta)(x)=x_{12} x_{13}$ and $(\hat{\delta} \otimes \iota)(y)=y_{13} y_{23}$. It is clear that, conversely, if $x$ and $y$ satisfy these relations, they give a representation, resp. a corepresentation of $W$.

In the same way as $C^{*}(G)$, we can define the universal $\mathrm{C}^{*}$-algebras $S_{\mathrm{u}}$ and $\hat{S}_{\mathrm{u}}$ for any l.c. quantum group (see [13]). There exists a universal corepresentation $W_{\mathrm{u}} \in \mathrm{M}\left(\hat{S} \otimes S_{\mathrm{u}}\right)$ such that

$$
S_{\mathrm{u}}=\left[(\omega \otimes \iota)\left(W_{\mathrm{u}}\right) \mid \omega \in \mathcal{L}(H)_{*}\right] .
$$

There is a bijective correspondence between representations $\pi: S_{\mathrm{u}} \rightarrow \mathcal{L}(K)$ of the C*-algebra $S_{\mathrm{u}}$ and corepresentations $y$ of $W$ on $K$ given by $y=(\iota \otimes \pi)\left(W_{\mathrm{u}}\right)$. All this is developed in [13].

When we want to stress the distinction between the reduced and the universal $\mathrm{C}^{*}$-algebras, we denote $S$ by $S_{\mathrm{r}}$.

The following definition is taken from page 482 in [2]. 
Definition 2.4. Let $W$ be a multiplicative unitary. A pair $(x, y)$ of a representation $x$ and a corepresentation $y$ of $W$ on the same Hilbert space $K$ is called covariant if

$$
y_{12} W_{13} x_{23}=x_{23} y_{12} .
$$

We remark that Proposition A.10 of [2] remains valid in the setting of l.c. quantum groups. Hence, if $W$ is the (left or right) regular representation of a l.c. quantum group and $(x, y)$ is a covariant pair, then $x$ is stably isomorphic to $W$ as well as $y$, but not jointly, and that is a very crucial point.

\section{c) Regularity and semi-regularity}

Let $V$ be a multiplicative unitary. Then, we have a naturally associated algebra (see [2]), defined by

$$
\mathcal{C}(V)=\left\{(\iota \otimes \omega)(\Sigma V) \mid \omega \in \mathcal{L}(H)_{*}\right\}
$$

We have $[\mathcal{C}(V) \mathcal{C}(V)]=[\mathcal{C}(V)]$, but $[\mathcal{C}(V)]$ is in general not a $\mathrm{C}^{*}$-algebra.

Next, we recall the notions of regularity [2] and semi-regularity [1] of a multiplicative unitary.

Definition 2.5. A multiplicative unitary $V$ is called regular if the closure of $\mathcal{C}(V)$ is $\mathcal{K}(H)$ and semi-regular if this closure contains $\mathcal{K}(H)$.

When $V$ is the regular representation of a l.c. quantum group, we have the following characterization of regularity and semi-regularity.

Proposition 2.6. Let $V$ be the right regular representation of a l.c. quantum group on the Hilbert space $H$. Then, $[\mathcal{C}(V)] \cong S \rtimes_{\mathrm{r}} \hat{S}$.

$S o, V$ is regular if and only if $S \rtimes_{\mathrm{r}} \hat{S}=\mathcal{K}(H)$ and $V$ is semi-regular if and only if $S \rtimes_{\mathrm{r}} \hat{S}$ contains $\mathcal{K}(H)$.

Remark that we consider $\delta$ as a right coaction of $(S, \delta)$ on the $\mathrm{C}^{*}$-algebra $S$. Then, the reduced crossed product $S \rtimes_{\mathrm{r}} \hat{S}$ is, by definition, given by $[\delta(S)(1 \otimes \hat{S})]$. Using the left regular representation, this last $\mathrm{C}^{*}$-algebra is isomorphic to $[S \hat{S}] \subset \mathcal{L}(H)$.

Proof. Suppose that $V$ is the right regular representation of $(M, \delta)$. From $[14,15]$, we know that we have two modular conjugations $J$ and $\hat{J}$ at our disposal: $J$ is the modular conjugation of the left invariant weight $\varphi$ and, up to a scalar, also the modular conjugation of the right invariant weight $\psi$, while $\hat{J}$ is the modular conjugation of the left invariant weight on the dual. We put $U=J \hat{J}$. Then, $U$ is a unitary and $U^{2}$ is scalar. From $[14,15]$, we know enough formulas to apply Proposition 6.9 of $[2]$ and to conclude that $(\Sigma(1 \otimes U) V)^{3}$ is scalar. Hence, up to a scalar, we have

$$
\Sigma V=(U \otimes 1) V^{*}(1 \otimes U) \Sigma V^{*} \Sigma(U \otimes 1) .
$$

Using the fact that $(J \otimes \hat{J}) V(J \otimes \hat{J})=V^{*}$ and the fact that $[(y \otimes 1) \hat{\delta}(x) \mid x, y \in \hat{S}]=\hat{S} \otimes \hat{S}$, we get

$$
\begin{aligned}
{[\mathcal{C}(V)] } & =\left[(\iota \otimes \omega)((1 \otimes J y J) \Sigma V(1 \otimes \hat{J} x \hat{J})) \mid x, y \in \hat{S}, \omega \in \mathcal{L}(H)_{*}\right] \\
& =\left[(\iota \otimes \omega)\left(\left(\Sigma(J \otimes \hat{J})(y \otimes 1) \hat{\delta}(x) V^{*}(J \otimes \hat{J})\right) \mid x, y \in \hat{S}, \omega \in \mathcal{L}(H)_{*}\right]=[\hat{J} \hat{S} \hat{J} \mathcal{C}(V)] .\right.
\end{aligned}
$$

Observing that $J \hat{S} J=\hat{S}$, we get that $[U \mathcal{C}(V) U]=[\hat{S} U \mathcal{C}(V) U]$. We combine this last equality with Equation (2.2) and the fact that $V \in \mathrm{M}(\hat{S} \otimes \mathcal{K}(H))$ to conclude that

$$
[U \mathcal{C}(V) U]=\left[(\iota \otimes \omega)\left((x \otimes k) V^{*}(1 \otimes U) \Sigma V^{*} \Sigma\right) \mid x \in \hat{S}, \omega \in \mathcal{L}(H)_{*}, k \in \mathcal{K}(H)\right]=[\hat{S} S] .
$$

Corollary 2.7. Let $V$ be the (left or right) regular representation of a l.c. quantum group on the Hilbert space $H$. Then, $[\mathcal{C}(V)]$ is always a $C^{*}$-algebra. 
Remark 2.8. The isomorphism $[\mathcal{C}(V)] \cong S \rtimes_{\mathrm{r}} \hat{S}$ is typical for the regular representation. Suppose that, on the Hilbert space $K,(x, y)$ is a covariant representation of the right regular representation $V$ of a l.c. quantum group (in the sense of Definition 2.4 below). Because both $x$ and $y$ are necessarily amplifications of the regular representation (individually, but not jointly, see the remark after Definition 2.4), this means that we have representations $\pi$ of $S$ and $\hat{\pi}$ of $\hat{S}$ on $K$ such that $x=(\hat{\pi} \otimes \iota)(V), y=(\iota \otimes \pi)(V)$ and which are covariant for the action $\delta$ of $(S, \delta)$ on $S$ in the sense that

$$
(\pi \otimes \iota) \delta(a)=x(\pi(a) \otimes 1) x^{*} \text { for all } a \in S .
$$

So, we have a representation of the full crossed product $S \rtimes_{\mathrm{f}} \hat{S}$, whose image is $[\pi(S) \hat{\pi}(\hat{S})]$.

On the other hand, we can write $\mathcal{V}=(\hat{\pi} \otimes \pi)(V)$, which is a multiplicative unitary on $K$. Then, the image of $S \rtimes_{\mathrm{f}} \hat{S}$ is equal to $\left[S_{\mathcal{V}} \hat{S}_{\mathcal{V}}\right]$. We claim that, $[\mathcal{C}(\mathcal{V})]$ is Morita equivalent to $[\mathcal{C}(V)] \cong S \rtimes_{\mathrm{r}} \hat{S}$. More precisely, $[\mathcal{C}(\mathcal{V})]$ is a $\mathrm{C}^{*}$-algebra and $[\mathcal{C}(\mathcal{V})] \otimes \mathcal{K}(K \otimes H)$ is spatially isomorphic to $[\mathcal{C}(V)] \otimes \mathcal{K}(K \otimes K)$. Hence, up to Morita equivalence, $[\mathcal{C}(\mathcal{V})]$ is always the same, while $\left[S_{\mathcal{V}} \hat{S}_{\mathcal{V}}\right]$ changes when $\mathcal{V}$ runs through the covariant images of $V$. Here, we can also observe that, if $V$ is semi-regular or regular, then the same holds for any covariant image $\mathcal{V}$ of $V$.

From the proofs of Proposition 3.19 and Corollaire 3.20 of [1], we know that the multiplicative unitaries $\mathcal{V}_{14} \mathcal{V}_{24}$ and $V_{36}$ on $K \otimes K \otimes H$ are unitarily equivalent. It follows that $[\mathcal{C}(V)] \otimes \mathcal{K}(K \otimes K) \cong\left[\mathcal{C}\left(\mathcal{V}_{13} \mathcal{V}_{23}\right)\right] \otimes \mathcal{K}(H)$. An easy calculation shows that $B:=\left[\mathcal{C}\left(\mathcal{V}_{13} \mathcal{V}_{23}\right)\right]=[\mathcal{C}(\mathcal{V}) \otimes \mathcal{K}(K)] \Sigma \mathcal{V}$. From the isomorphism above, we know that $B$ is a $\mathrm{C}^{*}$-algebra. Because $[(1 \otimes \mathcal{K}(K)) B]=B$ and $B$ is a $\mathrm{C}^{*}$-algebra, we have $B=[B(1 \otimes \mathcal{K}(K))]=$ $[\mathcal{C}(\mathcal{V}) \mathcal{C}(\mathcal{V})] \otimes \mathcal{K}(K)=[\mathcal{C}(\mathcal{V})] \otimes \mathcal{K}(K)$. Our claim is proven.

We make a more detailed study of regularity and semi-regularity of l.c. quantum groups in Section 5 .

\section{Bicrossed products of locally compact groups}

Definition 3.1. We call $G_{1}, G_{2}$ a matched pair of l.c. groups, if there is a given l.c. group $G$ such that $G_{1}$ and $G_{2}$ are closed subgroups of $G$ satisfying $G_{1} \cap G_{2}=\{e\}$ and such that $G_{1} G_{2}$ has complement of measure 0 in $G$.

Suppose, throughout this section, that we have fixed a matched pair $G_{1}, G_{2}$ of closed subgroups of $G$. We always use $g, h, k$ to denote elements in $G_{1}, s, t, r$ to denote elements in $G_{2}$ and accordingly, $d g, d s, \ldots$ to denote integration on $G_{1}, G_{2}$ with respect to a fixed right Haar measure. We use $x, y$ to denote elements in $G$ and $d x$ for the corresponding integration. The right Haar measure on $G$ is fixed such that the following proposition holds. We denote by $\Delta_{1}, \Delta_{2}$ and $\Delta$ the modular functions on $G_{1}, G_{2}$ and $G$.

Proposition 3.2. The map

$$
\theta: G_{1} \times G_{2} \rightarrow G: \theta(g, s)=g s
$$

preserves sets of measure zero and satisfies

$$
\int F(x) d x=\iint F(g s) \Delta_{1}(g) \Delta(g)^{-1} d g d s=\iint F(s g) \Delta_{2}(s) \Delta(s)^{-1} d g d s,
$$

for all positive Borel functions $F$ on $G$.

Proof. The proof of Lemma 4.10 in [19] can essentially be copied: if $K \in C_{c}\left(G_{1} \times G_{2}\right)$, we define the bounded, compactly supported, Borel function $\tilde{K}$ on $G$ by the formula $\tilde{K}(g s)=K(g, s) \Delta_{1}(g)^{-1} \Delta(g)$ and $\tilde{K}(x)=0$ for $x \in G \backslash G_{1} G_{2}$. Then, we define $I(K)=\int \tilde{K}(x) d x$. The same calculation as in [19] shows that $I$ is a right invariant integral. Because we supposed that $G$ is second countable, $G_{1} \times G_{2}$ is $\sigma$-compact and so, the integral $I$ must be non-zero. 
From the point of measure theory, we can not distinguish $G_{1} \times G_{2}$ and $G$. We define almost everywhere on $G$ the Borel functions $p_{1}: G \rightarrow G_{1}$ and $p_{2}: G \rightarrow G_{2}$ such that

$$
x=p_{1}(x) p_{2}(x) .
$$

Then, we can define

$$
\tau: L^{\infty}\left(G_{1}\right) \otimes L^{\infty}\left(G_{2}\right) \rightarrow L^{\infty}\left(G_{2}\right) \otimes L^{\infty}\left(G_{1}\right): \tau(F)(s, g)=F\left(p_{1}(s g), p_{2}(s g)\right) .
$$

The ${ }^{*}$-automorphism $\tau$ will be a (von Neumann algebraic version of an) inversion of $\left(L^{\infty}\left(G_{1}\right), \delta_{1}\right)$ and $\left(L^{\infty}\left(G_{2}\right), \delta_{2}\right)$ in the sense of [2], Definition 8.1. Denoting by $\sigma$ the flip map, it means that $\tau \sigma$ is a matching of $\left(L^{\infty}\left(G_{2}\right), \delta_{2}\right)$ and $\left(L^{\infty}\left(G_{1}\right), \delta_{1}^{\text {op }}\right)$ with trivial cocycles, in the sense of [19], Definition 2.1:

$$
(\tau \otimes \iota)(\iota \otimes \tau)\left(\delta_{1} \otimes \iota\right)=\left(\iota \otimes \delta_{1}\right) \tau \quad \text { and } \quad(\iota \otimes \tau)(\tau \otimes \iota)\left(\iota \otimes \delta_{2}\right)=\left(\delta_{2} \otimes \iota\right) \tau .
$$

Referring to Section 2.2 and in particular, Theorem 2.13 of [19], we can construct a l.c. quantum group using $\tau$. Because we have chosen to follow systematically the conventions of [2] and [3], we will state explicitly the needed formulas.

Define $\alpha: L^{\infty}\left(G_{1}\right) \rightarrow L^{\infty}\left(G_{2}\right) \otimes L^{\infty}\left(G_{1}\right)$ and $\beta: L^{\infty}\left(G_{2}\right) \rightarrow L^{\infty}\left(G_{2}\right) \otimes L^{\infty}\left(G_{1}\right)$ by

$$
\alpha(F)=\tau(F \otimes 1) \quad \text { and } \quad \beta(F)=\tau(1 \otimes F) .
$$

Then, $\alpha$ defines a left action of $G_{2}$ on $L^{\infty}\left(G_{1}\right)$ while $\beta$ defines a right action of $G_{1}$ on $L^{\infty}\left(G_{2}\right)$. In fact, if we consider $\tau \sigma$ as a matching of $\left(L^{\infty}\left(G_{2}\right), \delta_{2}\right)$ and $\left(L^{\infty}\left(G_{1}\right), \delta_{1}^{\text {op }}\right)$ in the sense of [19], these two actions $\alpha$ and $\beta$ precisely agree with the actions $\alpha$ and $\beta$ appearing in Definition 2.1 of [19]. We also have the obvious formulas

$$
\alpha(F)(s, g)=F\left(p_{1}(s g)\right) \text { and } \beta(F)(s, g)=F\left(p_{2}(s g)\right) .
$$

If we equip the quotient spaces $G / G_{2}$ and $G_{1} \backslash G$ with their canonical invariant measure class, the embedding $G_{1} \mapsto G / G_{2}$ identifies $G_{1}$ with a Borel subset of $G / G_{2}$ with complement of measure zero. Because of Proposition 3.2, this embedding, as well as its inverse, respects Borel sets of measure zero. Hence, it induces an isomorphism between $L^{\infty}\left(G_{1}\right)$ and $L^{\infty}\left(G / G_{2}\right)$. Then, $p_{1}$ can be considered as the projection of $G$ to $G / G_{2}$. Any $s \in G_{2}$ acts as a homeomorphism on $G / G_{2}$, preserving Borel sets of measure zero and hence, the map $p_{1}(s \cdot)$ is defined almost everywhere on $G_{1}$, preserves Borel sets of measure zero and gives precisely the automorphism $\alpha_{s}$ of $L^{\infty}\left(G_{1}\right)$. Similar considerations can be made for $\beta$.

We also conclude that, for a fixed $s \in G_{2}, s g \in G_{1} G_{2}$ for almost all $g \in G_{1}$. An analogous statement holds for a fixed $g \in G_{1}$. It follows that $p_{1}(s g)$ and $p_{2}(s g)$ are defined almost everywhere if either $s$ or $g$ is fixed. This will allow us to use freely $p_{1}(s g)$ and $p_{2}(s g)$ in integrals, see e.g. Equation (3.4).

We denote by $X$ and $Y$ the usual multiplicative unitaries on $H_{1}:=L^{2}\left(G_{1}\right)$ and $H_{2}:=L^{2}\left(G_{2}\right)$ respectively, defined by $(X \xi)(g, h)=\xi(g h, h)$ and $(Y \eta)(s, t)=\eta(s t, t)$. Then $\hat{Y}$ is the multiplicative unitary on $L^{2}\left(G_{2}\right)$ given by $(\hat{Y} \eta)(s, t)=\Delta_{2}(s)^{1 / 2} \eta\left(s, s^{-1} t\right)$.

Following [2] and [19], we can define the main actors of the paper.

Definition 3.3. Defining

$$
W=(\beta \otimes \iota)(\hat{Y})_{123}(\iota \otimes \alpha)(X)_{234},
$$

we get a multiplicative unitary $W$, which is the right regular representation of the l.c. quantum group $(M, \delta)$ given by

$$
M=\left(\alpha\left(L^{\infty}\left(G_{1}\right)\right) \cup \mathcal{L}\left(G_{2}\right) \otimes 1\right)^{\prime \prime}=G_{2} \ltimes_{\alpha} L^{\infty}\left(G_{1}\right) \quad \text { with } \quad \delta(z)=W(z \otimes 1) W^{*},
$$

where $\mathcal{L}\left(G_{2}\right)$ denotes the left regular representation of the group von Neumann algebra of $G_{2}$, generated by the unitaries $\lambda_{s}$ defined by $\left(\lambda_{s} \xi\right)(t)=\Delta_{2}(s)^{1 / 2} \xi\left(s^{-1} t\right)$.

The right Haar measure of $G_{1}$ is the left Haar measure of $G_{1}^{\text {op }}$ and secondly, using the modular function $\Delta_{2}$, the $L^{2}$-spaces of $G_{2}$ with right or left Haar measure are naturally isomorphic. Under this isomorphism $W$ agrees exactly with the $\hat{W}$ of [19], Definition 2.2 (one just has to interchange the indices 1 and 2 referring 
to $G_{1}$ and $G_{2}$ everywhere). Hence, the $(M, \delta)$ defined above agrees with $\left(M, \Delta^{\mathrm{op}}\right)$ in [19], where $\Delta^{\mathrm{op}}=\sigma \Delta$ with $\sigma$ the flip map. So, we get indeed that $W$ is the right regular representation of the l.c. quantum group $(M, \delta)$.

Dually, we have

$$
\hat{M}=\left(\beta\left(L^{\infty}\left(G_{2}\right)\right) \cup 1 \otimes \mathcal{R}\left(G_{1}\right)\right)^{\prime \prime}=L^{\infty}\left(G_{2}\right) \rtimes_{\beta} G_{1} \quad \text { with } \quad \hat{\delta}(z)=W^{*}(1 \otimes z) W,
$$

where $\mathcal{R}\left(G_{1}\right)$ denotes the right regular representation of the group von Neumann algebra of $G_{1}$, generated by the unitaries $\left(\rho_{g} \xi\right)(h)=\xi(h g)$.

Then, $(\hat{M}, \hat{\delta})$ above and $(\hat{M}, \hat{\Delta})$ in $[19]$ really agree.

From Equation (3.1), we get the following formulas for the comultiplication on the generators of $M$ and $\hat{M}$ :

$$
\begin{array}{ll}
\delta \alpha=(\alpha \otimes \alpha) \delta_{1} \quad \text { and } \quad(\iota \otimes \delta)\left(\hat{Y}_{12}\right)=\hat{Y}_{12}((\iota \otimes \alpha) \beta \otimes \iota)(\hat{Y})_{1234}, \\
\hat{\delta} \beta=(\beta \otimes \beta) \delta_{2} \quad \text { and } \quad(\hat{\delta} \otimes \iota)\left(X_{23}\right)=(\iota \otimes(\beta \otimes \iota) \alpha)(X)_{2345} X_{45} .
\end{array}
$$

Remark 3.4. If we interchange $G_{1}$ and $G_{2}$ and keep $G$, we also have a matched pair of l.c. groups. Performing the bicrossed product construction again, we get a multiplicative unitary on $L^{2}\left(G_{1} \times G_{2}\right)$, which through the unitary $(v \xi)(s, g)=\Delta_{1}(g)^{1 / 2} \Delta_{2}(s)^{1 / 2} \xi\left(g^{-1}, s^{-1}\right)$ corresponds to $\Sigma W^{*} \Sigma$. So, $S$ and $\hat{S}$ are interchanged and the comultiplications are flipped.

So, the von Neumann algebraic picture of the l.c. quantum group $(M, \delta)$ is completely clear. In this paper, we study the associated $\mathrm{C}^{*}$-algebras. To determine these, the following lemma will be crucial.

Lemma 3.5. Using the embedding $C_{0}\left(G / G_{2}\right) \rightarrow \mathrm{M}\left(C_{0}\left(G_{1}\right)\right) \subset L^{\infty}\left(G_{1}\right)$, we have

$$
\left[(\omega \otimes \iota) \alpha(F) \mid \omega \in \mathcal{L}\left(H_{2}\right)_{*}, F \in C_{0}\left(G_{1}\right)\right]=C_{0}\left(G / G_{2}\right) .
$$

Proof. We have to prove that for all $F_{i} \in C_{c}\left(G_{i}\right)$, the function

$$
H(g):=\int F_{1}\left(p_{1}(s g)\right) F_{2}(s) d s
$$

belongs to $C_{c}\left(G / G_{2}\right)$ and that these functions $H$ span a dense subspace of $C_{0}\left(G / G_{2}\right)$. It suffices to look at $F_{1}=\tilde{K}_{1} * P_{1}$ with $K_{1}, P_{1} \in C_{c}\left(G_{1}\right)$, i.e.

$$
F_{1}(g)=\int K_{1}(h) P_{1}(h g) d h .
$$

Take some $P_{2} \in C_{c}\left(G_{2}\right)$ such that $\int P_{2}\left(s^{-1}\right) d s=1$. On $G$, we define bounded Borel functions $K$ and $P$ with compact support by

$$
K(h s)=K_{1}(h) F_{2}(s) \Delta_{1}(h)^{-1} \Delta(h) \quad \text { and } \quad P(h s)=P_{1}(h) P_{2}(s)
$$

and such that $K$ and $P$ equal 0 outside $G_{1} G_{2}$. In particular, $K, P \in L^{2}(G)$ and defining $Q=\tilde{K} * P$, i.e.

$$
Q(x)=\int K(y) P(y x) d y,
$$

we have $Q \in C_{c}(G)$.

We claim that

$$
H(g)=\int Q\left(g t^{-1}\right) d t
$$


Take $g \in G_{1}$. Then, using Proposition 3.2,

$$
\begin{aligned}
\int Q\left(g t^{-1}\right) d t & =\iint K(y) P\left(y g t^{-1}\right) d y d t \\
& =\iiint K(h s) P\left(h s g t^{-1}\right) \Delta_{1}(h) \Delta(h)^{-1} d h d s d t \\
& =\iiint K_{1}(h) F_{2}(s) P_{1}\left(h p_{1}(s g)\right) P_{2}\left(p_{2}(s g) t^{-1}\right) d t d h d s \\
& =\iint K_{1}(h) F_{2}(s) P_{1}\left(h p_{1}(s g)\right) d h d s=H(g) .
\end{aligned}
$$

This proves our claim. We conclude that $H \in C_{c}\left(G / G_{2}\right)$. Moreover, the functions $H$ span a dense subset of $C_{0}\left(G / G_{2}\right)$, because in the proof above, the functions $K$ and $P$ both span a dense subset of $L^{2}(G)$, so that the functions $Q$ span a dense subset of $C_{0}(G)$.

From [19], we know that the multiplicative unitary $W$ is the (right) regular representation of the l.c. quantum group $(M, \delta)$. Hence, we have two associated $\mathrm{C}^{*}$-algebras:

$$
S=\left[(\omega \otimes \iota)(W) \mid \omega \in \mathcal{L}\left(H_{2} \otimes H_{1}\right)_{*}\right] \quad \text { and } \quad \hat{S}=\left[(\iota \otimes \omega)(W) \mid \omega \in \mathcal{L}\left(H_{2} \otimes H_{1}\right)_{*}\right] .
$$

The comultiplications $\delta$ and $\hat{\delta}$ restrict nicely to morphisms $\delta: S \rightarrow \mathrm{M}(S \otimes S)$ and $\hat{\delta}: \hat{S} \rightarrow \mathrm{M}(\hat{S} \otimes \hat{S})$.

As a first application of Lemma 3.5, we can identify $S$ and $\hat{S}$. We use $\lambda$ to denote the left regular representation of $C_{r}^{*}\left(G_{2}\right)$ on $L^{2}\left(G_{2}\right)$.

Proposition 3.6. The following equalities hold:

$$
\begin{aligned}
& S=G_{2} \ltimes_{\mathrm{r}} C_{0}\left(G / G_{2}\right)=\left[\left(\lambda\left(C_{r}^{*}\left(G_{2}\right)\right) \otimes 1\right) \alpha\left(C_{0}\left(G / G_{2}\right)\right)\right] \quad \text { and } \quad \alpha: C_{0}\left(G_{1}\right) \rightarrow \mathrm{M}(S) \quad \text { is non-degenerate, } \\
& \hat{S}=C_{0}\left(G_{1} \backslash G\right) \rtimes_{\mathrm{r}} G_{1}=\left[\left(1 \otimes C_{r}^{*}\left(G_{1}\right)\right) \beta\left(C_{0}\left(G_{1} \backslash G\right)\right)\right] \quad \text { and } \beta: C_{0}\left(G_{2}\right) \rightarrow \mathrm{M}(\hat{S}) \quad \text { is non-degenerate. }
\end{aligned}
$$

Proof. Observe that $\beta(F)=B(F \otimes 1) B^{*}$ for all $F \in L^{\infty}\left(G_{2}\right)$ where $B \in \mathrm{M}\left(\mathcal{K}\left(H_{2}\right) \otimes C_{0}\left(G_{1}\right)\right)$ is a representation of $X$, the canonical implementation of $\beta$. Because $B$ is a representation of $X$, we get

$$
\begin{aligned}
S & =\left[(\omega \otimes \mu \otimes \iota \otimes \iota)\left(\hat{Y}_{13}(\iota \otimes \iota \otimes \alpha)\left(B_{12}^{*} X_{23}\right)\right) \mid \omega \in \mathcal{L}\left(H_{2}\right)_{*}, \mu \in \mathcal{L}\left(H_{1}\right)_{*}\right] \\
& =\left[(\omega \otimes \mu \otimes \iota \otimes \iota)\left(\hat{Y}_{13}(\iota \otimes \iota \otimes \alpha)\left(B_{13} X_{23}\right)\right) \mid \omega \in \mathcal{L}\left(H_{2}\right)_{*}, \mu \in \mathcal{L}\left(H_{1}\right)_{*}\right] \\
& =\left[(\omega \otimes \iota \otimes \iota)\left(\hat{Y}_{12}(\iota \otimes \alpha)\left(B\left(1 \otimes C_{0}\left(G_{1}\right)\right)\right) \mid \omega \in \mathcal{L}\left(H_{2}\right)_{*}\right]=\left[\left(\lambda\left(C_{r}^{*}\left(G_{2}\right)\right) \otimes 1\right) \alpha\left(C_{0}\left(G_{1}\right)\right)\right] .\right.
\end{aligned}
$$

Because $S$ is a $\mathrm{C}^{*}$-algebra and because of Lemma 3.5, we get

$$
S=\left[\left(\lambda\left(C_{r}^{*}\left(G_{2}\right)\right) \otimes 1\right) \alpha\left(C_{0}\left(G_{1}\right)\right)\left(\lambda\left(C_{r}^{*}\left(G_{2}\right)\right) \otimes 1\right)\right]=\left[\left(\lambda\left(C_{r}^{*}\left(G_{2}\right)\right) \otimes 1\right) \alpha\left(C_{0}\left(G / G_{2}\right)\right)\left(\lambda\left(C_{r}^{*}\left(G_{2}\right)\right) \otimes 1\right)\right] .
$$

By definition, $G_{2} \ltimes_{\mathrm{r}} C_{0}\left(G / G_{2}\right)=\left[\left(\lambda\left(C_{r}^{*}\left(G_{2}\right)\right) \otimes 1\right) \alpha\left(C_{0}\left(G / G_{2}\right)\right)\right]$ and this is a $\mathrm{C}^{*}$-algebra. Hence, $S=$ $G_{2} \ltimes_{\mathrm{r}} C_{0}\left(G / G_{2}\right)$. So, we have proven the first line of the statement above. The second line is analogous (or follows by interchanging $G_{1}$ and $G_{2}$ and applying Remark 3.4).

Next, we prove the corresponding result for the universal $\mathrm{C}^{*}$-algebras.

Proposition 3.7. Suppose that $\mathcal{W}$ is a corepresentation of $W$ on a Hilbert space $K$.

a) There exist unique corepresentations $y$ of $\hat{Y}$ and $x$ of $X$ on $K$ such that

$$
\mathcal{W}=(\beta \otimes \iota)(y) x_{23} .
$$

b) If we denote by $\pi_{1}$ and $\hat{\pi}_{2}$ the representations of $C_{0}\left(G_{1}\right)$ and $C^{*}\left(G_{2}\right)$ corresponding to $x$ and $y$ and if we define the representation $\tilde{\pi}_{1}$ of $C_{0}\left(G / G_{2}\right)$ using the extension of $\pi_{1}$ to $\mathrm{M}\left(C_{0}\left(G_{1}\right)\right)$, then the pair $\left(\tilde{\pi}_{1}, \hat{\pi}_{2}\right)$ is a covariant representation for the left action of $G_{2}$ on $C_{0}\left(G / G_{2}\right)$. 
c) The representation $\pi$ of $S_{\mathrm{u}}$ associated to $\mathcal{W}$ and the representation $\tilde{\pi}_{1} \times \hat{\pi}_{2}$ of $G_{2} \ltimes_{\mathrm{f}} C_{0}\left(G / G_{2}\right)$ have the same image.

Conversely, every covariant representation for the left action of $G_{2}$ on $G / G_{2}$ is obtained in this way from a corepresentation $\mathcal{W}$ of $W$.

In particular,

$$
S_{\mathrm{u}} \cong G_{2} \ltimes_{\mathrm{f}} C_{0}\left(G / G_{2}\right)
$$

in a natural way. An analogous statement holds for $\hat{S}_{\mathrm{u}}$ and in particular, $\hat{S}_{\mathrm{u}} \cong C_{0}\left(G_{1} \backslash G\right) \rtimes_{\mathrm{f}} G_{1}$.

Proof. a) This follows from [4]. Observe that $x \in \mathrm{M}\left(C_{r}^{*}\left(G_{1}\right) \otimes \mathcal{K}(K)\right)$ and $y \in \mathrm{M}\left(C_{0}\left(G_{2}\right) \otimes \mathcal{K}(K)\right)$.

b) Because $\mathcal{W}$ is a corepresentation, we get the following equality in $\mathcal{L}\left(H_{2} \otimes H_{1} \otimes H_{2} \otimes H_{1} \otimes K\right)$.

$$
(\hat{\delta} \otimes \iota)\left(x_{23}\right)=(\beta \otimes \iota)\left(y^{*}\right)_{345} x_{25}(\beta \otimes \iota)(y)_{345} x_{45} .
$$

Define the non-degenerate representation $\tilde{\pi}_{1}$ of $C_{0}\left(G / G_{2}\right)$ as in the statement of the proposition.

We know that, in $\mathcal{L}\left(H_{2} \otimes H_{1} \otimes H_{2} \otimes H_{1} \otimes H_{1}\right)$,

$$
(\hat{\delta} \otimes \iota)\left(X_{23}\right)=(\iota \otimes(\beta \otimes \iota) \alpha)(X)_{2345} X_{45}
$$

From Proposition 3.6, it follows that $X_{23} \in \mathrm{M}\left(\hat{S} \otimes C_{0}\left(G_{1}\right)\right)$ and so, it follows that $(\iota \otimes(\beta \otimes \iota) \alpha)(X)_{2345} \in$ $\mathrm{M}\left(\hat{S} \otimes \hat{S} \otimes C_{0}\left(G_{1}\right)\right)$, from which we conclude that

$$
(\beta \otimes \iota) \alpha: C_{0}\left(G_{1}\right) \rightarrow \mathrm{M}\left(\hat{S} \otimes C_{0}\left(G_{1}\right)\right)
$$

is a well-defined and non-degenerate *-homomorphism. From Equation (3.7) and the fact that $\left(\iota \otimes \pi_{1}\right)(X)=$ $x$, we conclude that, in $\mathcal{L}\left(H_{2} \otimes H_{1} \otimes H_{2} \otimes H_{1} \otimes K\right)$,

$$
(\hat{\delta} \otimes \iota)\left(x_{23}\right)=\left(\iota \otimes\left(\iota \otimes \iota \otimes \pi_{1}\right)(\beta \otimes \iota) \alpha\right)(X)_{2345} x_{45} .
$$

Combining with Equation (3.6), we find that

$$
\left(\iota \otimes\left(\iota \otimes \iota \otimes \pi_{1}\right)(\beta \otimes \iota) \alpha\right)(X)=(\beta \otimes \iota)\left(y^{*}\right)_{234} x_{14}(\beta \otimes \iota)(y)_{234},
$$

which yields in $\mathcal{L}\left(H_{2} \otimes H_{1} \otimes K\right)$ the equality

$$
\left(\iota \otimes \iota \otimes \pi_{1}\right)(\beta \otimes \iota) \alpha(F)=(\beta \otimes \iota)\left(y^{*}\right)\left(1 \otimes 1 \otimes \pi_{1}(F)\right)(\beta \otimes \iota)(y)
$$

for all $F \in C_{0}\left(G_{1}\right)$. Because of non-degenerateness, the same holds for all $F \in \mathrm{M}\left(C_{0}\left(G_{1}\right)\right)$ and hence, for $F \in C_{0}\left(G / G_{2}\right)$. As $G_{2}$ acts continuously on $C_{0}\left(G / G_{2}\right)$, we have $\alpha: C_{0}\left(G / G_{2}\right) \rightarrow \mathrm{M}\left(C_{0}\left(G_{2}\right) \otimes C_{0}\left(G / G_{2}\right)\right)$ and so, we arrive at the formula

$$
\left(\iota \otimes \tilde{\pi}_{1}\right) \alpha(F)=y^{*}\left(1 \otimes \tilde{\pi}_{1}(F)\right) y,
$$

for all $F \in C_{0}\left(G / G_{2}\right)$. This precisely gives the required covariance of $\left(\tilde{\pi}_{1}, \hat{\pi}_{2}\right)$.

c) Denote by $\pi$ and $\tilde{\pi}_{1} \times \hat{\pi}_{2}$ the corresponding representations of $S_{\mathrm{u}}$ and $G_{2} \ltimes_{\mathrm{f}} C_{0}\left(G / G_{2}\right)$. We have to prove that they have the same image and this will be analogous to the proof of Proposition 3.6. We use again the canonical implementation $B \in \mathrm{M}\left(\mathcal{K}\left(H_{2}\right) \otimes C_{0}\left(G_{1}\right)\right)$ of $\beta$. Observe that

$\pi\left(S_{\mathrm{u}}\right)=\left[(\omega \otimes \mu \otimes \iota)(\mathcal{W}) \mid \omega \in \mathcal{L}\left(H_{2}\right)_{*}, \mu \in \mathcal{L}\left(H_{1}\right)_{*}\right]=\left[(\omega \otimes \mu \otimes \iota)\left(y_{13} B_{12}^{*} x_{23}\right) \mid \omega \in \mathcal{L}\left(H_{2}\right)_{*}, \mu \in \mathcal{L}\left(H_{1}\right)_{*}\right]$.

Because $B_{12} B_{13} X_{23}=X_{23} B_{12}$, we get $B_{12}^{*} x_{23}=\left(\iota \otimes \pi_{1}\right)(B)_{13} x_{23} B_{12}^{*}$ and hence,

$$
\begin{aligned}
\pi\left(S_{\mathrm{u}}\right) & =\left[(\omega \otimes \mu \otimes \iota)\left(y_{13}\left(\iota \otimes \pi_{1}\right)(B)_{13} x_{23}\right) \mid \omega \in \mathcal{L}\left(H_{2}\right)_{*}, \mu \in \mathcal{L}\left(H_{1}\right)_{*}\right] \\
& =\left[(\omega \otimes \iota)\left(y\left(\iota \otimes \pi_{1}\right)(B) \pi_{1}\left(C_{0}\left(G_{1}\right)\right) \mid \omega \in \mathcal{L}\left(H_{2}\right)_{*}\right]=\left[\hat{\pi}_{2}\left(C^{*}\left(G_{2}\right)\right) \pi_{1}\left(C_{0}\left(G_{1}\right)\right)\right] .\right.
\end{aligned}
$$

From Equation (3.10), it follows that

$$
\tilde{\pi}_{1}((\omega \beta \otimes \iota) \alpha(F))=(\omega \otimes \iota)\left((\beta \otimes \iota)\left(y^{*}\right)\left(1 \otimes 1 \otimes \pi_{1}(F)\right)(\beta \otimes \iota)(y)\right)
$$


for all $F \in C_{0}\left(G_{1}\right)$ and $\omega \in \mathcal{L}\left(H_{2} \otimes H_{1}\right)_{*}$. Combining this with Lemma 3.5, it follows that

$$
\left[\hat{\pi}_{2}\left(C^{*}\left(G_{2}\right)\right) \tilde{\pi}_{1}\left(C_{0}\left(G / G_{2}\right)\right) \hat{\pi}_{2}\left(C^{*}\left(G_{2}\right)\right)\right]=\left[\hat{\pi}_{2}\left(C^{*}\left(G_{2}\right)\right) \pi_{1}\left(C_{0}\left(G_{1}\right)\right) \hat{\pi}_{2}\left(C^{*}\left(G_{2}\right)\right)\right] .
$$

The image of $\tilde{\pi}_{1} \times \hat{\pi}_{2}$ is given by $\left[\hat{\pi}_{2}\left(C^{*}\left(G_{2}\right)\right) \pi_{1}\left(C_{0}\left(G / G_{2}\right)\right)\right]$ and is a $\mathrm{C}^{*}$-algebra. Also $\pi\left(S_{\mathrm{u}}\right)$ is a $\mathrm{C}^{*}$-algebra, so that the calculation above shows that, indeed $\pi$ and $\tilde{\pi}_{1} \times \hat{\pi}_{2}$ have the same image. This concludes the proof of the first part of the proposition.

Suppose now, conversely, that we have a covariant representation $\left(\tilde{\pi}_{1}, \hat{\pi}_{2}\right)$ on $K$ for the left action of $G_{2}$ on $G / G_{2}$. Denote by $y$ the corepresentation of $\hat{Y}$ corresponding to $\hat{\pi}_{2}$. The representation $\tilde{\pi}_{1}$ defines a measure class on $G / G_{2}$ which, by covariance, is invariant under the action of $G_{2}$. We claim that this measure class is supported by the image of $G_{1}$ in $G / G_{2}$. By invariance, the transformation $(s, \bar{x}) \mapsto(s, \overline{s x})$ of $G_{2} \times G / G_{2}$ preserves sets of measure zero and so, we have to prove that the set of pairs $(s, \bar{x})$ such that $\overline{s x} \notin G_{1}$ has measure zero. Using the Fubini theorem, it is enough to prove that, for any $x \in G$, the set of all $s \in G_{2}$ such that $\overline{s x} \notin G_{1}$ has measure zero. But this last set is equal to the set of all $s \in G_{2}$ such that $g s \notin G_{1} G_{2} x^{-1}$ for all $g \in G_{1}$. And here, we get a set of measure zero and our claim has been proved.

Because the measure class on $G / G_{2}$ is supported by $G_{1}$, we can use the Borel calculus to find a nondegenerate representation $\pi_{1}$ of $C_{0}\left(G_{1}\right)$ on $K$ such that $\tilde{\pi}_{1}$ is indeed obtained by extending $\pi_{1}$ to $\mathrm{M}\left(C_{0}\left(G_{1}\right)\right.$ ). Put $x=\left(\iota \otimes \pi_{1}\right)(X)$. We have to prove that $\mathcal{W}:=(\beta \otimes \iota)(y) x_{23}$ is a corepresentation of $W$. Because $\tilde{\pi}_{1}$ makes sense on all bounded Borel functions on $G / G_{2}$ (which is essentially the same thing as bounded Borel functions on $G_{1}$ ), we get, by covariance

$$
\tilde{\pi}_{1}\left(F\left(p_{1}(s \cdot)\right)\right)=y_{s}^{*} \tilde{\pi}_{1}(F) y_{s},
$$

for all bounded Borel functions on $F$ on $G_{1}$. Integrating, we conclude that

$$
\tilde{\pi}_{1}((\omega \otimes \iota) \alpha(F))=(\omega \otimes \iota)\left(y^{*}\left(1 \otimes \tilde{\pi}_{1}(F)\right) y\right),
$$

for all bounded Borel functions on $F$ on $G_{1}$ and $\omega \in \mathcal{L}\left(H_{2}\right)_{*}$. From this, it follows that Equation (3.10) holds for all $F \in C_{0}\left(G_{1}\right)$ and as we saw above, this yields that $\mathcal{W}$ is a corepresentation of $W$.

Observe that it follows immediately that the projection *-homomorphism of $S_{\mathrm{u}}$ onto $S$ corresponds exactly to the projection *-homomorphism of the full crossed product $G_{2} \ltimes_{\mathrm{f}} C_{0}\left(G / G_{2}\right)$ onto the reduced crossed product $G_{2} \ltimes_{\mathrm{r}} C_{0}\left(G / G_{2}\right)$. From the proof of the previous proposition, it also follows that we have a non-degenerate *-homomorphism $C_{0}\left(G_{1}\right) \rightarrow \mathrm{M}\left(S_{\mathrm{u}}\right)$.

Next, we consider covariant representations and we identify $S \rtimes_{\mathrm{r}, \mathrm{f}} \hat{S}$.

Proposition 3.8. Suppose that $\mathcal{V}$ is a representation and $\mathcal{W}$ is a corepresentation of $W$, such that $(\mathcal{V}, \mathcal{W})$ is a covariant representation of $W$.

Take the unique corepresentations $x$ of $X$ and $y$ of $\hat{Y}$ and the unique representations a of $X$ and $b$ of $\hat{Y}$ such that

$$
\mathcal{V}=b_{12}(\iota \otimes \alpha)(a) \quad \text { and } \quad \mathcal{W}=(\beta \otimes \iota)(y) x_{23}
$$

Denote by $\pi_{1}$ and $\pi_{2}$ the representations of $C_{0}\left(G_{1}\right)$ and $C_{0}\left(G_{2}\right)$ associated with $x$ and $b$, respectively.

a) The ranges of $\pi_{1}$ and $\pi_{2}$ commute and we can define a non-degenerate representation $\pi$ of $C_{0}\left(G_{1} \times G_{2}\right)$. Extending first to $C_{b}\left(G_{1} \times G_{2}\right)$ and then restricting to $C_{0}(G)$ using the map $(g, s) \mapsto g s$, we get a nondegenerate representation $\tilde{\pi}$ of $C_{0}(G)$.

b) The unitaries $a_{g}$ and $y_{s}$ commute for all $g \in G_{1}, s \in G_{2}$.

c) The pair $\left(\tilde{\pi},\left(y_{s} a_{g}\right)_{(s, g)}\right)$ is a covariant representation for the action of $G_{2} \times G_{1}$ on $C_{0}(G)$, where $G_{2}$ acts on the left and $G_{1}$ on the right.

d) The images of the associated representations of $S \rtimes_{\mathrm{f}} \hat{S}$ and $\left(G_{2} \times G_{1}\right) \ltimes_{\mathrm{f}} C_{0}(G)$ coincide. 
Conversely, every covariant representation for the action of $G_{2} \times G_{1}$ on $C_{0}(G)$ is obtained in this way from a covariant pair for $W$.

In particular,

$$
S \rtimes_{\mathrm{f}} \hat{S} \cong\left(G_{2} \times G_{1}\right) \ltimes_{\mathrm{f}} C_{0}(G)
$$

in a natural way.

Proof. Let $(\mathcal{V}, \mathcal{W})$ be a covariant pair for $W$ on $K$ and take, using Proposition 3.7, $x, y, a$ and $b$ as in the statement of the proposition. As we remarked right after Definition 2.4, both $\mathcal{W}$ and $\mathcal{V}$ are stably isomorphic to $W$, but not jointly. This means that we have faithful, normal *-homomorphisms $\eta: M \rightarrow \mathcal{L}(K)$ and $\hat{\eta}: \hat{M} \rightarrow \mathcal{L}(K)$ such that $\mathcal{W}=(\iota \otimes \eta)(W)$ and $\mathcal{V}=(\hat{\eta} \otimes \iota)(W)$. Restricting to the von Neumann subalgebras $\alpha\left(L^{\infty}\left(G_{1}\right)\right)$ and $\mathcal{L}\left(G_{2}\right) \otimes 1$ of $M$ and $\beta\left(L^{\infty}\left(G_{2}\right)\right)$ and $1 \otimes \mathcal{R}\left(G_{1}\right)$ of $\hat{M}$, we obtain faithful, normal *-homomorphisms $\pi_{1}, \hat{\pi}_{2}, \pi_{2}$ and $\hat{\pi}_{1}$ respectively, such that

$$
x=\left(\iota \otimes \pi_{1}\right)(X), \quad y=\left(\iota \otimes \hat{\pi}_{2}\right)(\hat{Y}), \quad a=\left(\hat{\pi}_{1} \otimes \iota\right)(X) \quad \text { and } \quad b=\left(\pi_{2} \otimes \iota\right)(\hat{Y}) .
$$

The covariance of the pair $(\mathcal{V}, \mathcal{W})$ is equivalent to each of the following formulas:

$$
(\eta \otimes \iota) \delta(z)=\mathcal{V}(\eta(z) \otimes 1) \mathcal{V}^{*} \quad \text { for all } z \in M \quad(\iota \otimes \hat{\eta}) \hat{\delta}(z)=\mathcal{W}^{*}(1 \otimes \hat{\eta}(z)) \mathcal{W} \quad \text { for all } z \in \hat{M}
$$

Evaluating these formulas on the generators of $M$ and $\hat{M}$ following Equations (3.2) and (3.3), covariance is equivalent to each of the following two lines of formulas:

$$
\begin{aligned}
& x_{12}(\iota \otimes \alpha)(X)_{134}=\mathcal{V}_{234} x_{12} \mathcal{V}_{234}^{*} \quad, \quad y_{12}\left(\left(\iota \otimes \pi_{1}\right) \beta \otimes \iota\right)(\hat{Y})_{123}=\mathcal{V}_{234} y_{12} \mathcal{V}_{234}^{*}, \\
& (\beta \otimes \iota)(\hat{Y})_{124} b_{34}=\mathcal{W}_{123}^{*} b_{34} \mathcal{W}_{123} \quad, \quad\left(\iota \otimes\left(\pi_{2} \otimes \iota\right) \alpha\right)(X)_{234} a_{34}=\mathcal{W}_{123}^{*} a_{34} \mathcal{W}_{123}
\end{aligned}
$$

Using the explicit expression of $\mathcal{V}$, the second formula of Equation (3.11) becomes

$$
b_{23}^{*} y_{12}\left(\left(\iota \otimes \pi_{1}\right) \beta \otimes \iota\right)(\hat{Y})_{123} b_{23}=(\iota \otimes \iota \otimes \alpha)\left(a_{23} y_{12} a_{23}^{*}\right) \text {. }
$$

Because $\alpha\left(L^{\infty}\left(G_{1}\right)\right) \cap \mathcal{L}\left(G_{2}\right) \otimes 1=\mathbb{C}$, we find a unitary $v \in \mathcal{L}\left(H_{2} \otimes K\right)$, such that $a_{23} y_{12} a_{23}^{*}=v_{12}$. Treating in the same way the second formula of Equation (3.12), we find that $y_{12}^{*} a_{23} y_{12} \in 1 \otimes \mathcal{L}\left(K \otimes H_{1}\right)$. But, $y_{12}^{*} a_{23} y_{12}=y_{12}^{*} v_{12} a_{23}$, which yields the existence of $u \in \mathcal{L}(K)$ such that $v=y(1 \otimes u)$. Because clearly both $v$ and $y$ are corepresentations of $\hat{Y}$, we find that $u=1$. Hence, we arrived at the commutation of $a_{23}$ and $y_{12}$. This means that the unitaries $a_{g}$ and $y_{s}$ commute for all $g \in G_{1}, s \in G_{2}$.

Next, we observe that $b^{*}(1 \otimes F) b=\left(\pi_{2} \otimes \iota\right) \delta_{2}(F)$ for all $F \in L^{\infty}\left(G_{2}\right)$. Combining this with the formula $\left(\delta_{2} \otimes \iota\right) \alpha=(\iota \otimes \alpha) \alpha$, we can rewrite the first formula of Equation (3.11) in the form

$$
b_{23}^{*} x_{12} b_{23}=(\iota \otimes \iota \otimes \alpha)\left(a_{23} x_{12} a_{23}^{*}\left(\iota \otimes\left(\pi_{2} \otimes \iota\right) \alpha\right)\left(X^{*}\right)\right) \text {. }
$$

As above, it follows that $b_{23}^{*} x_{12} b_{23} \in \mathcal{L}\left(H_{1} \otimes K\right) \otimes 1$. Treating similarly the first formula of Equation (3.12), we find

$$
x_{23} b_{34} x_{23}^{*}=(\beta \otimes \iota \otimes \iota)\left(\left(\left(\iota \otimes \pi_{1}\right) \beta \otimes \iota\right)\left(\hat{Y}^{*}\right) y_{12}^{*} b_{23} y_{12}\right)
$$

and hence, that $x_{12} b_{23} x_{12}^{*} \in 1 \otimes \mathcal{L}\left(K \otimes H_{2}\right)$. A similar reasoning as above yields now the commutation of $x_{12}$ and $b_{23}$. This means that the ranges of $\pi_{1}$ and $\pi_{2}$ commute. Combining this with Equation (3.15), we get

$$
\left(\left(\iota \otimes \pi_{1}\right) \beta \otimes \iota\right)(\hat{Y}) b_{23}=y_{12}^{*} b_{23} y_{12}
$$

Denote by $\pi$ the non-degenerate representation of $C_{0}\left(G_{1} \times G_{2}\right)$ on $K$ such that $\pi\left(F_{1} \otimes F_{2}\right)=\pi_{1}\left(F_{1}\right) \pi_{2}\left(F_{2}\right)$. Dualizing the continuous map $(g, s) \mapsto g s$, we embed $C_{0}(G)$ into $\mathrm{M}\left(C_{0}\left(G_{1} \times G_{2}\right)\right)$ and obtain the nondegenerate representation $\tilde{\pi}$ of $C_{0}(G)$ on $K$. We have to prove that $\left(\tilde{\pi},\left(a_{g} y_{s}\right)\right)$ is a covariant representation.

We claim that

$$
\gamma:=((\beta \otimes \iota) \tau \otimes \iota)\left(\iota \otimes \delta_{2}\right): C_{0}\left(G_{1} \times G_{2}\right) \rightarrow \mathrm{M}\left(\mathcal{K}\left(H_{2} \otimes H_{1}\right) \otimes C_{0}\left(G_{1} \times G_{2}\right)\right)
$$


is well-defined and non-degenerate. It suffices to verify this statement on $C_{0}\left(G_{1}\right) \otimes 1$ and $1 \otimes C_{0}\left(G_{2}\right)$ separately. For $C_{0}\left(G_{1}\right) \otimes 1$, it follows from Equation (3.8). On the other hand, we observe that

$$
(\gamma \otimes \iota)\left(\hat{Y}_{23}\right)=B_{12} B_{13} \hat{Y}_{15} B_{13}^{*} B_{12}^{*} \hat{Y}_{45} \in \mathrm{M}\left(\mathcal{K}\left(H_{2} \otimes H_{1}\right) \otimes C_{0}\left(G_{1} \times G_{2}\right) \otimes \mathcal{K}\left(H_{2}\right)\right),
$$

where $B \in \mathrm{M}\left(\mathcal{K}\left(H_{2}\right) \otimes C_{0}\left(G_{1}\right)\right)$ is again the canonical implementation of $\beta$. This proves our claim. Using this last equation and Equation (3.16), we also observe that

$$
\begin{aligned}
(\iota \otimes \iota \otimes \pi \otimes \iota)(\gamma \otimes \iota)(1 \otimes \hat{Y}) & =B_{12}\left(\left(\iota \otimes \pi_{1}\right) \beta \otimes \iota\right)(\hat{Y})_{134} B_{12}^{*} b_{34} \\
& =(\beta \otimes \iota \otimes \iota)\left(y_{12}^{*} b_{23} y_{12}\right)=(\beta \otimes \iota)\left(y^{*}\right)_{123}(\pi \otimes \iota)(1 \otimes \hat{Y})_{34}(\beta \otimes \iota)(y)_{123} .
\end{aligned}
$$

Combining this with Equation (3.10), which holds because $\mathcal{W}$ is a corepresentation of $W$, we get

$$
(\iota \otimes \iota \otimes \pi) \gamma(F)=(\beta \otimes \iota)\left(y^{*}\right)(1 \otimes 1 \otimes \pi(F))(\beta \otimes \iota)(y),
$$

for all $F \in C_{0}\left(G_{1} \times G_{2}\right)$. By non-degenerateness, the same holds for $F \in C_{0}(G)$, which exactly means that

$$
\tilde{\pi}(F(s \cdot))=y_{s}^{*} \tilde{\pi}(F) y_{s}
$$

for all $F \in C_{0}(G)$ and $s \in G_{2}$.

One similarly proves that $\tilde{\pi}(F(\cdot g))=a_{g} \tilde{\pi}(F) a_{g}^{*}$ for all $F \in C_{0}(G)$ and $g \in G_{1}$. So, $\left(\tilde{\pi},\left(a_{g} y_{s}\right)\right)$ is a covariant representation.

Denote the image of the representation of $S \rtimes_{\mathrm{f}} \hat{S}$ associated with $(\mathcal{V}, \mathcal{W})$ by $A$. Then, by definition

$$
A=\left[(\iota \otimes \omega)(\mathcal{V})(\mu \otimes \iota)(\mathcal{W}) \mid \omega, \mu \in \mathcal{L}\left(H_{2} \otimes H_{1}\right)_{*}\right] .
$$

It follows from Proposition 3.7 and its proof that

$$
A=\left[\hat{\pi}_{2}\left(C^{*}\left(G_{2}\right) \pi_{1}\left(C_{0}\left(G_{1}\right)\right) \pi_{2}\left(C_{0}\left(G_{2}\right)\right) \hat{\pi}_{1}\left(C^{*}\left(G_{1}\right)\right)\right]=\left[\hat{\pi}_{2}\left(C^{*}\left(G_{2}\right) \pi\left(C_{0}\left(G_{1} \times G_{2}\right)\right) \hat{\pi}_{1}\left(C^{*}\left(G_{1}\right)\right)\right] .\right.\right.
$$

From Equation (3.17), we know that

$$
\pi\left((\omega \otimes \iota \otimes \iota)(\tau \otimes \iota)\left(\iota \otimes \delta_{2}\right)(F)\right)=(\omega \otimes \iota)\left(y^{*}(1 \otimes \pi(F)) y\right)
$$

for all $F \in C_{0}\left(G_{1} \times G_{2}\right)$ and $\omega \in \mathcal{L}\left(H_{2}\right)_{*}$. Lemma 3.9 following this proof, tells us that the left hand side spans a dense subset of $\tilde{\pi}\left(C_{0}(G)\right)$. So, we get

$$
\left[\hat { \pi } _ { 2 } \left(C^{*}\left(G_{2}\right) \tilde{\pi}\left(C_{0}(G)\right) \hat{\pi}_{1}\left(C^{*}\left(G_{1}\right)\right]=\left[\hat { \pi } _ { 2 } \left(C^{*}\left(G_{2}\right) \pi\left(C_{0}\left(G_{1} \times G_{2}\right)\right) \hat{\pi}_{1}\left(C^{*}\left(G_{1}\right)\right] .\right.\right.\right.\right.
$$

Because both $A$ and the image of $\left(G_{2} \times G_{1}\right) \ltimes_{\mathrm{f}} C_{0}(G)$ are $\mathrm{C}^{*}$-algebras, we easily get that $A$ indeed equals the image of $\left(G_{2} \times G_{1}\right) \ltimes_{\mathrm{f}} C_{0}(G)$.

Suppose now that, conversely, we have a covariant representation $\left(\tilde{\pi},\left(y_{s} a_{g}\right)\right)$ for the action of $G_{2} \times G_{1}$ on $C_{0}(G)$. The representation $\tilde{\pi}$ gives rise to a measure class on $G$ which is invariant by multiplication on the left by $G_{2}$ and on the right by $G_{1}$. We claim that this measure class is supported by $G_{1} G_{2}$. Because the transformation $(s, x) \mapsto(s, s x)$ on $G_{2} \times G$ preserves Borel sets of measure zero, it is enough to prove that for any $x \in G$, the set of all $s \in G_{2}$ such that $s x \notin G_{1} G_{2}$ has measure zero. But this is the case, as we already saw in the proof of Proposition 3.7.

Hence, the Borel functional calculus provides a non-degenerate representation $\pi$ of $C_{0}\left(G_{1} \times G_{2}\right)$ such that $\tilde{\pi}$ is obtained by extending $\pi$ to $\mathrm{M}\left(C_{0}\left(G_{1} \times G_{2}\right)\right)$ and then restricting to $C_{0}(G)$ through the map $(g, s) \mapsto g s$. We denote by $\pi_{1}$ and $\pi_{2}$ the corresponding representations of $C_{0}\left(G_{1}\right)$ and $C_{0}\left(G_{2}\right)$. Then, we can define $x=\left(\iota \otimes \pi_{1}\right)(X)$ and $b=\left(\pi_{2} \otimes \iota\right)(\hat{Y})$. Defining

$$
\mathcal{V}=b_{12}(\iota \otimes \alpha)(a) \text { and } \mathcal{W}=(\beta \otimes \iota)(y) x_{23},
$$

it follows from Proposition 3.7 that $\mathcal{V}$ is a representation of $W$ and $\mathcal{W}$ is a corepresentation of $W$. 
We know that $\tilde{\pi}(F(s \cdot))=y_{s}^{*} \tilde{\pi}(F) y_{s}$ for all $s \in G_{2}$ and all bounded Borel functions $F$ on $G$. Integrating, it follows in particular that

$$
\tilde{\pi}\left((\omega \otimes \iota \otimes \iota)(\beta \otimes \iota) \delta_{2}(F)\right)=(\omega \otimes \iota)\left(y^{*}\left(1 \otimes \pi_{2}(F)\right) y\right)
$$

for all $\omega \in \mathcal{L}\left(H_{2}\right)_{*}$ and all $F \in C_{0}\left(G_{2}\right)$. Evaluating this formula on $F=(\iota \otimes \mu)(\hat{Y})$, we get

$$
\left(\left(\iota \otimes \pi_{1}\right) \beta \otimes \iota\right)(\hat{Y}) b_{23}=y_{12}^{*} b_{23} y_{12},
$$

which makes sense because $\beta: C_{0}\left(G_{2}\right) \rightarrow \mathrm{M}\left(\mathcal{K}\left(H_{2}\right) \otimes C_{0}\left(G_{1}\right)\right)$ is well-defined and non-degenerate (using the canonical implementation of $\beta$ ). Because $a_{23}$ and $y_{12}$ commute, it follows that Equation (3.13) holds and hence, also the second formula of Equation (3.11) holds.

Because $\tilde{\pi}(F(\cdot g))=a_{g} \tilde{\pi}(F) a_{g}^{*}$ for all $g \in G_{1}$ and all bounded Borel functions $F$ on $G$, we find similarly that

$$
\tilde{\pi}\left((\iota \otimes \iota \otimes \omega)(\iota \otimes \alpha) \delta_{1}(F)\right)=(\iota \otimes \omega)\left(a\left(\pi_{1}(F) \otimes 1\right) a^{*}\right)
$$

for all $\omega \in \mathcal{L}\left(H_{1}\right)_{*}$ and all $F \in C_{0}\left(G_{1}\right)$, which yields, in the same way,

$$
x_{12}\left(\iota \otimes\left(\pi_{2} \otimes \iota\right) \alpha\right)(X)=a_{23} x_{12} a_{23}^{*},
$$

where $\left(\pi_{2} \otimes \iota\right) \alpha$ makes again sense using the canonical implementation of $\alpha$. Because $x_{12}$ and $b_{23}$ commute, we get that Equation (3.14) holds and hence, also the first formula in Equation (3.11). Combining both formulas of Equation (3.11) and the definitions of $\mathcal{V}, \mathcal{W}$ and $W$, we get

$$
\begin{aligned}
\mathcal{V}_{345} \mathcal{W}_{123} \mathcal{V}_{345}^{*} & =(\beta \otimes \iota)(y)_{123}\left(\left(\iota \otimes \iota \otimes \pi_{1}\right)\left(\iota \otimes \delta_{1}\right) \beta \otimes \iota\right)(\hat{Y})_{1234} x_{23}(\iota \otimes \alpha)(X)_{245} \\
& =\mathcal{W}_{123}(\beta \otimes \iota)(\hat{Y})_{124}(\iota \otimes \alpha)(X)_{245}=\mathcal{W}_{123} W_{1245}
\end{aligned}
$$

where we used the formula $\left(\iota \otimes \pi_{1}\right) \delta_{1}(F)=x(F \otimes 1) x^{*}$ for all $F \in C_{0}\left(G_{1}\right)$. So, we precisely get that $(\mathcal{V}, \mathcal{W})$ is a covariant pair for $W$.

The following lemma was needed to prove the previous proposition. It is completely analogous to Lemma 3.5 above.

Lemma 3.9. Using the map $(g, s) \mapsto g s$, we embed $C_{0}(G) \rightarrow \mathrm{M}\left(C_{0}\left(G_{1} \times G_{2}\right)\right) \subset L^{\infty}\left(G_{1} \times G_{2}\right)$. Then, we have

$$
C_{0}(G)=\left[(\omega \otimes \iota \otimes \iota)(\tau \otimes \iota)\left(\iota \otimes \delta_{2}\right)\left(C_{0}\left(G_{1} \times G_{2}\right)\right) \mid \omega \in \mathcal{L}\left(H_{2}\right)_{*}\right] .
$$

Proof. We have to prove that the functions

$$
g t \mapsto \int K_{2}(s) F_{1}\left(p_{1}(s g)\right) F_{2}\left(p_{2}(s g) t\right) d s
$$

belongs to $C_{c}(G)$ whenever $F_{1} \in C_{c}\left(G_{1}\right), K_{2}, F_{2} \in C_{c}\left(G_{2}\right)$ and that they span a dense subset of $C_{0}(G)$. It suffices to consider $F_{1}$ of the form $\tilde{K}_{1} * P_{1}$ with $K_{1}, P_{1} \in C_{c}\left(G_{1}\right)$ and

$$
F_{1}(g)=\int K_{1}(h) P_{1}(h g) d h .
$$

Then, we can define bounded Borel functions $K$ and $P$ with compact support on $G$ by the formulas

$$
P(g s)=P_{1}(g) F_{2}(s) \quad, \quad K(g s)=K_{1}(g) K_{2}(s) \Delta_{1}(g)^{-1} \Delta(g)
$$

and $P$ and $K$ equal 0 outside $G_{1} G_{2}$. Because $K$ and $P$ belong to $L^{2}(G)$ and have compact support, the function $H:=K * P$ defined by

$$
H(x)=\int K(y) P(y x) d y
$$


belongs to $C_{c}(G)$. But, using Proposition 3.2,

$$
\begin{aligned}
H(g t) & =\int K(y) P(y g t) d y=\iint K(h s) P(h s g t) \Delta_{1}(h) \Delta(h)^{-1} d h d s \\
& =\iint K_{1}(h) K_{2}(s) P_{1}\left(h p_{1}(s g)\right) F_{2}\left(p_{2}(s g) t\right) d h d s=\int K_{2}(s) F_{1}\left(p_{1}(s g)\right) F_{2}\left(p_{2}(s g) t\right) d s .
\end{aligned}
$$

This ends the proof, because it is clear that the functions $K$ and $P$ span dense subspaces of $L^{2}(G)$ and hence the functions $H$ span a dense subspace of $C_{0}(G)$.

We also characterize the reduced crossed products in the following easily proved proposition.

Proposition 3.10. The regular covariant pair $(W, W)$ for $W$ corresponds through the procedure of Proposition 3.8 to a representation of $\left(G_{2} \times G_{1}\right) \ltimes_{\mathrm{f}} C_{0}(G)$ which is stably isomorphic to the regular representation. In particular,

$$
S \rtimes_{\mathrm{r}} \hat{S} \cong\left(G_{2} \times G_{1}\right) \ltimes_{\mathrm{r}} C_{0}(G)
$$

and the projection *-homomorphisms from the full onto the reduced crossed products are intertwined by the isomorphisms $S \rtimes_{\mathrm{f}, \mathrm{r}} \hat{S} \cong\left(G_{2} \times G_{1}\right) \ltimes_{\mathrm{f}, \mathrm{r}} C_{0}(G)$.

Proof. Because the orbit of $e$ under the action of $G_{2} \times G_{1}$, which is $G_{2} G_{1}$, is dense in $G$, it follows that the covariant representation associated with this orbit, on the Hilbert space $L^{2}\left(G_{2} \times G_{1}\right)$, is stably isomorphic to the regular representation of $\left(G_{2} \times G_{1}\right) \ltimes_{\mathrm{f}} C_{0}(G)$. It is clear that the $\mathcal{V}$ and $\mathcal{W}$ corresponding to this covariant representation are twice $W$.

Using this proposition, we prove our main theorem.

Theorem 3.11. The multiplicative unitary $W$ of the bicrossed product l.c. quantum group $(M, \delta)$ is regular if and only if the map

$$
\theta: G_{1} \times G_{2} \rightarrow G: \theta(g, s)=g s
$$

is a homeomorphism of $G_{1} \times G_{2}$ onto $G$. The multiplicative unitary $W$ is semi-regular if and only if $\theta$ is a homeomorphism of $G_{1} \times G_{2}$ onto an open subset of $G$ with complement of measure zero.

Proof. In the proof of the previous proposition, we have seen that $S \rtimes_{\mathrm{r}} \hat{S}$ is precisely given by the image, denoted by $A$, of the irreducible representation of $\left(G_{2} \times G_{1}\right) \ltimes_{\mathrm{f}} C_{0}(G)$ corresponding to the free orbit $G_{2} G_{1}$. From Lemma 3.12, it follows that $A=\mathcal{K}\left(H_{2} \otimes H_{1}\right)$ if and only if this orbit is closed and homeomorphic to $G_{2} \times G_{1}$ and that $\mathcal{K}\left(H_{2} \otimes H_{1}\right) \subset A$ if and only if this orbit is locally closed and homeomorphic to $G_{2} \times G_{1}$. Because the orbit is dense and because of Proposition 2.6, we precisely arrive at the statement of the theorem, using also that $G_{1} G_{2}=\left(G_{2} G_{1}\right)^{-1}$.

In the next section, we will give examples were the image of $\theta$ is not open and hence, the associated multiplicative unitary is not semi-regular. Nevertheless, it should be observed that $W$, being the regular representation of a l.c. quantum group, is always manageable in the sense of [21]. So, not all manageable multiplicative unitaries are semi-regular.

The next lemma is well known (see [8] for a related result), but we include a short proof for completeness.

Lemma 3.12. Suppose that a l.c. group $G$ acts continuously (on the right) on a l.c. space $X$. Let $x_{0} \in X$ have a free orbit, i.e. the map $\theta: G \rightarrow X: \theta(g)=x_{0} \cdot g$ is injective.

Denote by $\pi$ the representation of $C_{0}(X) \rtimes_{\mathrm{f}} G$ on $L^{2}(G)$ corresponding to the orbit of $x_{0}$. Then,

a) The image of $\pi$ contains $\mathcal{K}\left(L^{2}(G)\right)$ if and only if the orbit $\theta(G)$ is locally closed and $\theta$ is a homeomorphism of $G$ onto $\theta(G)$.

b) The image of $\pi$ is equal to $\mathcal{K}\left(L^{2}(G)\right)$ if and only if the orbit $\theta(G)$ is closed and $\theta$ is a homeomorphism of $G$ onto $\theta(G)$. 
Proof. Suppose first that the image of $\pi$ contains $\mathcal{K}\left(L^{2}(G)\right)$. Equip $\theta(G)$ with its relative topology and assume that $\theta^{-1}$ is not continuous. Then, we find elements $g_{n} \in G$ such that $g_{n}(n \geq 1)$ remains outside a neighborhood of $g_{0}$, but $x_{0} \cdot g_{n} \rightarrow x_{0} \cdot g_{0}$. Consider the dense subalgebra of the image of $\pi$ consisting of the operators $\gamma(F), F \in C_{c}(G \times X)$, defined by

$$
(\gamma(F) \xi)(g)=\int_{G} F\left(h, x_{0} \cdot g\right) \xi(g h) d h .
$$

Take a function $\eta \in L^{2}(G)$ with $\|\eta\|=1$ and with small enough support such that $\left\langle\lambda_{g_{n}} \eta, \lambda_{g_{0}} \eta\right\rangle=0$ for all $n \geq 1$, where $\left(\lambda_{g}\right)$ is the left regular representation of $G$. One verifies immediately that

$$
\left\langle\lambda_{g_{n}} \eta, \gamma(F) \lambda_{g_{n}} \eta\right\rangle \rightarrow\left\langle\lambda_{g_{0}} \eta, \gamma(F) \lambda_{g_{0}} \eta\right\rangle \quad \text { for all } \quad F \in C_{c}(G \times X)
$$

Hence, the same holds for all $a \in \pi\left(C_{0}(X) \rtimes_{\mathrm{f}} G\right)$ instead of $\gamma(F)$ and, in particular, for all $a \in \mathcal{K}\left(L^{2}(G)\right)$. This gives a contradiction when we take for $a$ the projection on $\lambda_{g_{0}} \eta$. So, $\theta^{-1}$ is continuous. This means that $\theta(G)$ is locally compact in its relative topology, which precisely means that $\theta(G)$ is locally closed in $X$.

Suppose next that the image of $\pi$ is precisely $\mathcal{K}\left(L^{2}(G)\right)$. Denote by $Y$ the closure of $\theta(G)$. From the previous paragraph, we already know that $\theta(G)$ is open in $Y$ and that $\theta$ is a homeomorphism. Suppose that $\theta(G) \neq Y$, take $x_{1} \in Y \backslash \theta(G)$ and take $g_{n} \in G$ such that $x_{0} \cdot g_{n} \rightarrow x_{1}$. Then, $g_{n}$ goes to infinity. Writing $\pi_{1}$ for the representation of $C_{0}(X) \rtimes_{\mathrm{f}} G$ on $L^{2}(G)$ corresponding to the orbit of $x_{1}$, whose image contains the dense subalgebra of operators $\gamma_{1}(F), F \in C_{c}(G \times X)$, we observe that

$$
\left\langle\lambda_{g_{n}} \eta, \gamma(F) \lambda_{g_{n}} \eta\right\rangle \rightarrow\left\langle\eta, \gamma_{1}(F) \eta\right\rangle \quad \text { for all } \quad \eta \in L^{2}(G), F \in C_{c}(G \times X) .
$$

But, $\lambda_{g_{n}} \eta \rightarrow 0$ weakly, as $g_{n} \rightarrow \infty$. Because the image of $\pi$ is supposed to be exactly the compact operators, it follows from the previous formula that $\left\langle\eta, \gamma_{1}(F) \eta\right\rangle=0$ for all $F$ and $\eta$. This is a contradiction.

The converse implications are easy to prove.

Using the theory that we developed so far, it is also easy to give examples of l.c. quantum groups such that the projection *-homomorphism from $S \rtimes_{\mathrm{f}} \hat{S}$ onto $S \rtimes_{\mathrm{r}} \hat{S}$ is not faithful. Loosely speaking, this means that the action of $(S, \delta)$ on itself by translation is not amenable and in particular, not proper (whatever this means).

Proposition 3.13. Suppose that $G_{1}$ and $G_{2}$ are conjugated, i.e. there exists an element $z_{0} \in G$ such that $G_{2}=z_{0} G_{1} z_{0}^{-1}$.

a) $S \rtimes_{\mathrm{f}, \mathrm{r}} \hat{S}$ is strongly Morita equivalent with $S_{\mathrm{u}, \mathrm{r}}$.

b) The projection *-homomorphism from $S \rtimes_{\mathrm{f}} \hat{S}$ onto $S \rtimes_{\mathrm{r}} \hat{S}$ is faithful if and only if $G_{1}$ is amenable.

c) $M \cong \hat{M} \cong \mathcal{L}\left(L^{2}\left(G_{1}\right)\right)$.

Proof. a) We use an argument which is essentially contained in Rieffel's paper [17], but we include a sketch of it for completeness. Write $B=C_{0}\left(G / G_{1}\right)$. On $C_{c}(G)$, the continuous compactly supported functions on $G$, we define a $B$-valued inner product by

$$
\langle\xi, \eta\rangle(x)=\int_{G_{1}}(\bar{\xi} \eta)\left(x g^{-1}\right) d g
$$

Completion yields the Hilbert $B$-module $\mathcal{E}$. Because the right action of $G_{1}$ on $G$ is proper, there is only one crossed product $C_{0}(G) \rtimes G_{1}$, which can be identified with $\mathcal{K}(\mathcal{E})$, the 'compact' operators on the Hilbert $B$-module $\mathcal{E}$. Because $\mathcal{E}$ is full, we have a Morita equivalence between $C_{0}(G) \rtimes G_{1}$ and $B$. Then, we have

$$
S \rtimes_{\mathrm{f}, \mathrm{r}} \hat{S} \cong\left(G_{2} \times G_{1}\right) \ltimes_{\mathrm{f}, \mathrm{r}} C_{0}(G) \cong G_{2} \ltimes_{\mathrm{f}, \mathrm{r}} \mathcal{K}(\mathcal{E}) \cong \mathcal{K}\left(G_{2} \ltimes_{\mathrm{f}, \mathrm{r}} \mathcal{E}\right),
$$

where all isomorphisms are natural and intertwine the projection of full onto reduced crossed products and where $G_{2} \ltimes_{\mathrm{f}, \mathrm{r}} \mathcal{E}$ is the obvious full Hilbert $G_{2} \ltimes_{\mathrm{f}, \mathrm{r}} C_{0}\left(G / G_{1}\right)$-module. In the identifications above, only the 
isomorphism $G_{2} \ltimes_{\mathrm{f}} \mathcal{K}(\mathcal{E}) \cong \mathcal{K}\left(G_{2} \ltimes_{\mathrm{f}} \mathcal{E}\right)$ requires some care: consider the $\mathrm{C}^{*}$-algebra $\mathcal{K}(\mathcal{E} \oplus B)$ in which $\mathcal{K}(\mathcal{E})$ is a full corner and on which $G_{2}$ acts by automorphisms. Then, $G_{2} \ltimes_{\mathrm{f}} \mathcal{K}(\mathcal{E})$ is a full corner of $G_{2} \ltimes_{\mathrm{f}} \mathcal{K}(\mathcal{E} \oplus B)$ and is as such identified with $\mathcal{K}\left(G_{2} \ltimes_{\mathrm{f}} \mathcal{E}\right)$.

Observing that the right multiplication by $z_{0}^{-1}$ gives a homeomorphism of $G / G_{1}$ onto $G / G_{2}$ intertwining the left action of $G_{2}$, we see that $G_{2} \ltimes_{\mathrm{f}, \mathrm{r}} C_{0}\left(G / G_{1}\right) \cong S_{\mathrm{u}, \mathrm{r}}$. So, we have proven the required strong Morita equivalence.

b) Because of the Morita equivalence above, the projection of $S \rtimes_{\mathrm{f}} \hat{S}$ onto $S \rtimes_{\mathrm{r}} \hat{S}$ is faithful if and only if the projection of $S_{\mathrm{u}}$ onto $S$ is faithful. From Theorem 15 in [6], it follows that this last projection is faithful if and only if $G_{2}$ is amenable. Because of conjugacy, this is equivalent to the amenability of $G_{1}$.

c) As above, we observe that the left action of $G_{2}$ on $G / G_{2}$ is isomorphic to the left action of $G_{2}$ on $G / G_{1}$. Using the isomorphism $L^{\infty}\left(G / G_{1}\right) \cong L^{\infty}\left(G_{2}\right)$, we see that the action of $G_{2}$ on $L^{\infty}\left(G / G_{2}\right)$ is isomorphic to the left translation of $G_{2}$ on $L^{\infty}\left(G_{2}\right)$. Hence, $M \cong \mathcal{L}\left(L^{2}\left(G_{2}\right)\right)$.

Remark 3.14. Although in the situation of Proposition 3.13, we have a strong Morita equivalence between $S \rtimes_{\mathrm{f}} \hat{S}$ and $S_{\mathrm{u}}$ and hence, an isomorphism $S \rtimes_{\mathrm{f}} \hat{S} \otimes \mathcal{K} \cong S_{\mathrm{u}} \otimes \mathcal{K}$, this isomorphism is very much 'twisted' for the following reason: if $G_{1}$ is non-amenable, $S_{\mathrm{u}}$ is very different from $S$, but nevertheless, we claim that, for any l.c. quantum group $(M, \delta)$, we have a natural, injective ${ }^{*}$-homomorphism $M \rightarrow \mathrm{M}\left(S \rtimes_{\mathrm{f}} \hat{S}\right)$. As usual we write $S$ and $\hat{S}$ for the underlying $\mathrm{C}^{*}$-algebras as in Equation (2.1).

From the remark after Definition 2.4, it follows that we can realize (in a natural way) $S \rtimes_{\mathrm{f}} \hat{S}$ on a Hilbert space $K$ such that there exist normal, faithful ${ }^{*}$-homomorphisms $\pi: M \rightarrow \mathcal{L}(K)$ and $\hat{\pi}: \hat{M} \rightarrow \mathcal{L}(K)$ such that $S \rtimes_{\mathrm{f}} \hat{S}=[\pi(S) \hat{\pi}(\hat{S})]$. We prove that $\pi(M) \subset \mathrm{M}\left(S \rtimes_{\mathrm{f}} \hat{S}\right)$. Take $x \in S^{\prime \prime}$ and observe that

$$
(\hat{\pi} \otimes \iota)(V)(\pi(x) \otimes 1)=(\pi \otimes \iota) \delta(x)(\hat{\pi} \otimes \iota)(V)=(\pi \otimes \iota)\left(W^{*}\right)(1 \otimes x)(\pi \otimes \iota)(W)(\hat{\pi} \otimes \iota)(V),
$$

where $V \in \mathrm{M}(\hat{S} \otimes \mathcal{K})$ is the right regular representation of $(M, \delta)$ and $W \in \mathrm{M}(S \otimes \mathcal{K})$ is the left regular representation. Hence,

$$
\begin{aligned}
\left(S \rtimes_{\mathrm{f}} \hat{S}\right) \pi(x) & =\left[\pi(S)(\iota \otimes \omega)\left((\pi \otimes \iota)\left(W^{*}\right)(1 \otimes x)(\pi \otimes \iota)(W)(\hat{\pi} \otimes \iota)(V)\right) \mid \omega \in \mathcal{L}(H)_{*}\right] \\
& =\left[(\iota \otimes \omega)((\pi(S) \otimes x)(\pi \otimes \iota)(W)(\hat{\pi} \otimes \iota)(V)) \mid \omega \in \mathcal{L}(H)_{*}\right] \subset S \rtimes_{\mathrm{f}} \hat{S} .
\end{aligned}
$$

So, we are done.

\section{Examples}

We start off by presenting a fairly general example. Suppose that $\mathcal{A}$ is a l.c. ring, with unit, but not necessarily commutative and $\mathcal{A} \neq\{0\}$. Denote by $\mathcal{A}^{\star}$ the group of invertible elements in $\mathcal{A}$. Then, $\mathcal{A}^{\star}$ is a l.c. group by considering it as the closed subspace $\{(a, b) \mid a b=1=b a\}$ of $\mathcal{A} \times \mathcal{A}$.

Next, we can define the $a x+b$-group on this l.c. $\operatorname{ring} \mathcal{A}$.

$$
G=\mathcal{A}^{\star} \times \mathcal{A} \quad \text { with } \quad(a, x)(b, y)=(a b, x+a y) .
$$

Defining the closed subgroups (isomorphic with $\mathcal{A}^{\star}$ )

$$
G_{1}=\left\{(a, a-1) \mid a \in \mathcal{A}^{\star}\right\} \quad \text { and } \quad G_{2}=\left\{(b, 0) \mid b \in \mathcal{A}^{\star}\right\},
$$

we observe that $G_{1} G_{2}$ consists of all pairs $(a, x)$ such that $x+1 \in \mathcal{A}^{\star}$. Hence, we get a matched pair of l.c. groups if and only if $\mathcal{A}^{\star}$ has complement of (additive) Haar measure zero in $\mathcal{A}$. Observe that $G_{1}$ and $G_{2}$ are conjugated by the element $(-1,-1) \in G$.

Suppose that $\mathcal{A}$ is a l.c. ring, different from $\{0\}$, with $\mathcal{A} \backslash \mathcal{A}^{*}$ of (additive) Haar measure zero.

Denote the bicrossed product multiplicative unitary associated to the above matched pair by $\mathcal{W}_{\mathcal{A}}$ and the associated l.c. quantum group by $(M, \delta)_{\mathcal{A}}$.

Proposition 4.1. We have the following properties. 
- The multiplicative unitary $\mathcal{W}_{\mathcal{A}}$ is not regular. It is semi-regular if and only if $\mathcal{A}^{\star}$ is open in $\mathcal{A}$.

- The projection of $S \rtimes_{\mathrm{f}} \hat{S}$ onto $S \rtimes_{\mathrm{r}} \hat{S}$ is faithful if and only if the l.c. group $\mathcal{A}^{\star}$ is amenable.

- The dual $(\hat{M}, \hat{\delta})_{\mathcal{A}}$ is isomorphic to the opposite quantum group $\left(M, \delta^{\mathrm{op}}\right)_{\mathcal{A}}$.

- The associated $C^{*}$ - and von Neumann algebras are given by

$$
\begin{aligned}
& S_{\mathrm{u}, \mathrm{r}} \cong \hat{S}_{\mathrm{u}, \mathrm{r}} \cong \mathcal{A}^{\star} \ltimes_{\mathrm{f}, \mathrm{r}} C_{0}(\mathcal{A}) \quad \text { where } \mathcal{A}^{\star} \text { multiplies } \mathcal{A} \text { on the left }, \\
& M \cong \hat{M} \cong \mathcal{L}\left(L^{2}\left(\mathcal{A}^{\star}\right)\right) \\
& S \rtimes_{\mathrm{f}, \mathrm{r}} \hat{S} \cong \mathcal{K}\left(L^{2}\left(\mathcal{A}^{\star}\right)\right) \otimes\left(\mathcal{A}^{\star} \ltimes_{\mathrm{f}, \mathrm{r}} C_{0}(\mathcal{A})\right) .
\end{aligned}
$$

Proof. Using Theorem 3.11 and Proposition 3.13, we get immediately the first two statements of the proposition.

To prove the third statement, define the unitary $\mathcal{U}$ on $L^{2}\left(\mathcal{A}^{\star} \times \mathcal{A}^{\star}\right)$ by the formula

$$
(\mathcal{U} \xi)(b, a)=\Delta_{\mathcal{A}^{\star}}(a b)^{1 / 2} \xi\left(a^{-1}, b^{-1}\right),
$$

where $\Delta_{\mathcal{A}^{\star}}$ is the modular function of $\mathcal{A}^{\star}$. A straightforward calculation on the generators shows that $\mathcal{U} M \mathcal{U}^{*}=\hat{M}$ and that this isomorphism intertwines $\delta^{\text {op }}$ on $M$ and $\hat{\delta}$ on $\hat{M}$

There is a homeomorphism from $G / G_{2}$ onto $\mathcal{A}$ mapping $\overline{(a, x)}$ to $x$, intertwining the left action of $G_{2}$ on $G / G_{2}$ with the left multiplication of $\mathcal{A}^{\star}$ on $\mathcal{A}$. So, using Propositions 3.6 and 3.7, we get $S_{\mathrm{u}, \mathrm{r}} \cong \mathcal{A}^{\star} \ltimes_{\mathrm{f}, \mathrm{r}} C_{0}(\mathcal{A})$ where $\mathcal{A}^{\star}$ multiplies $\mathcal{A}$ on the left. Because of the already proven third statement, we have $S_{\mathrm{u}, \mathrm{r}} \cong \hat{S}_{\mathrm{u}, \mathrm{r}}$ and so, we have proven the first formula of the fourth statement.

The second formula of the fourth statement follows from Proposition 3.13.

Finally, using the homeomorphism $(d, x) \mapsto(d, x-d)$ of $G$, the action of $G_{2} \times G_{1}$ on $G$ is isomorphic to the action $b \cdot(d, x) \cdot a=(b d a, b x)$ of $G_{2} \times G_{1}$ on $G$. If we take the crossed product with the action of $a \in \mathcal{A}^{\star}$, we obtain $\mathcal{K}\left(L^{2}\left(\mathcal{A}^{\star}\right)\right) \otimes C_{0}(\mathcal{A})$, on which $\mathcal{A}^{\star}$ acts diagonally. This diagonal action is isomorphic with the amplified action on $C_{0}(\mathcal{A})$ and we obtain the third formula, using Propositions 3.8 and 3.10 .

Observe that the von Neumann algebraic picture of $(M, \delta)_{\mathcal{A}}$ is fairly trivial, because $M \cong \mathcal{L}\left(L^{2}\left(\mathcal{A}^{\star}\right)\right)$. Nevertheless, the $\mathrm{C}^{*}$-algebra picture is far from trivial. As we will see in concrete examples below, the $\mathrm{C}^{*}$-algebra $S$ need not be type I.

An example of a non-amenable $\mathcal{A}^{*}$ is, for instance, given by $\mathcal{A}=M_{2}(\mathbb{R})$. In that case the projection of $S \rtimes_{\mathrm{f}} \hat{S}$ onto $S \rtimes_{\mathrm{r}} \hat{S}$ is not an isomorphism.

Example 4.2. Let $\mathcal{P}$ be an infinite set of prime numbers such that

$$
\sum_{p \in \mathcal{P}} \frac{1}{p}<\infty
$$

Define

$$
\mathcal{A}=\prod_{p \in \mathcal{P}}^{\prime} \mathbb{Q}_{p},
$$

where $\mathbb{Q}_{p}$ is the l.c. field of $p$-adic numbers and where the prime means that we take the restricted Cartesian product: we only consider elements $\left(x_{p}\right)$ that eventually belong to the compact sub-ring $\mathbb{Z}_{p}$ of $p$-adic integers. When we equip $\mathcal{A}$ with the usual l.c. topology, we obtain a l.c. ring such that $\mathcal{A}^{\star}$ has complement of measure zero in $\mathcal{A}$ but $\mathcal{A}^{\star}$ has empty interior in $\mathcal{A}$. The fact that $\mathcal{A}^{\star}$ has complement of measure zero follows from a straightforward application of the Borel-Cantelli lemma: normalizing the Haar measure on $\mathbb{Q}_{p}$ such that $\mathbb{Z}_{p}$ has measure one, we observe that $\mathbb{Z}_{p} \backslash \mathbb{Z}_{p}^{\star}$ has measure $\frac{1}{p}$, which is assumed to be summable over $p \in \mathcal{P}$.

Observe that $\mathcal{A}$ is a (restricted) ring of adeles and that $S_{\mathcal{A}} \cong \mathcal{A}^{\star} \ltimes C_{0}(\mathcal{A})$. Such a $\mathrm{C}^{*}$-algebra $S_{\mathcal{A}}$ is not of type I and is intensively studied by J.B. Bost and A. Connes in [5] $]^{1}$.

\footnotetext{
${ }^{1}$ More precisely, they study the Hecke algebra, which is $p\left(\mathcal{A}^{\star} \ltimes C_{0}(\mathcal{A})\right) p$ for a natural projection $p$.
} 
Remark 4.3. Observe that in the previous example, we can replace $\mathbb{Q}_{p}$ by the field of formal Laurent series over the finite field $F_{p}$ with $p$ elements. We have the compact sub-ring of formal power series over $F_{p}$ and can perform an analogous construction.

Example 4.4. Another, more canonical example can be given, much in the same spirit as Example 4.2. Denote for every prime number $p$ by $K_{p}$ the (unique) non-ramified extension of degree 2 of $\mathbb{Q}_{p}$. Let $\mathcal{O}\left(K_{p}\right)$ be the compact ring of integers in $K_{p}$. Because the extension is non-ramified, the Haar measure of $\mathcal{O}\left(K_{p}\right) \backslash$ $\mathcal{O}\left(K_{p}\right)^{\star}$ is $\frac{1}{p^{2}}$. Because this sequence is summable, we can take

$$
\mathcal{A}=\prod_{p \text { prime }}^{\prime} K_{p}
$$

Remark 4.5. Consider the easiest case $\mathcal{A}=\mathbb{R}$. Define $K=L^{2}\left(\mathbb{R}^{*}\right)$. A covariant representation on the Hilbert space $K$ for $G_{2} \times G_{1}$ acting on $C_{0}(G)$ is given by

$$
\pi(F)(x)=F(x, x) \quad \text { for } F \in C_{0}(G), x \in \mathbb{R}^{*} \quad \text { and } \quad\left(\lambda_{b} \rho_{a}\right)_{(b, a) \in G_{2} \times G_{1}} .
$$

We can then take the covariant image of the bicrossed product multiplicative unitary $W_{\mathbb{R}}$ and this yields the multiplicative unitary $\mathcal{W}$ on $K$ given by

$$
(\mathcal{W} \xi)(x, y)=\xi\left(\frac{x y}{x+y+1}, \frac{y}{x+1}\right) .
$$

Observing that $\left(\mathcal{W}^{*} \xi\right)(x, y)=\xi\left(\frac{x(y+1)}{y}, x+y+x y\right)$, we see that both $\mathcal{W}$ and $\mathcal{W}^{*}$ leave $L^{2}\left(\mathbb{R}_{+}^{*}\right) \otimes L^{2}\left(\mathbb{R}_{+}^{*}\right)$ invariant. So, the restriction of $\mathcal{W}$ gives us a multiplicative unitary $\tilde{\mathcal{W}}$ on $K_{+}:=L^{2}\left(\mathbb{R}_{+}^{*}\right)$. It is easy to check that the weak closure of $S_{\tilde{\mathcal{W}}}$ consists precisely of all the operators $T \in \mathcal{L}\left(K_{+}\right)$such that $P_{[x,+\infty} K_{+}$is an invariant subspace of $T$ for all $x \in \mathbb{R}_{+}^{*}$. Hence, this weak closure is not invariant under involution. This means that $S_{\tilde{\mathcal{W}}}$ is not a $\mathrm{C}^{*}$-algebra. Analogously, $\hat{S}_{\tilde{\mathcal{W}}}$ is not a $\mathrm{C}^{*}$-algebra.

The multiplicative unitary $\tilde{\mathcal{W}}$ is very singular and should certainly not be considered as the multiplicative unitary of a quantum group.

Using the transformation $v:] 0,1\left[\rightarrow R_{+}^{*}: v(x)=\frac{x}{1-x}\right.$, the multiplicative unitary $\tilde{\mathcal{W}}$ is transformed to a multiplicative unitary $X$ on $L^{2}(] 0,1[, d \mu)$ given by

$$
(X \xi)(x, y)=\xi\left(x y, y \frac{1-x}{1-x y}\right) .
$$

The transformation $v(x, y)=\left(x y, y \frac{1-x}{1-x y}\right)$ is studied in [12] and is shown to be essentially the only pentagonal transformation on $] 0,1[$ of the form $v(x, y)=(x y, u(x, y))$ with $u$ continuously differentiable.

We can construct an even more singular multiplicative unitary $Y$ on the Hilbert space $l^{2}\left(\mathbb{Q}_{+}^{*}\right)$ which is formally given by the same formula as $\tilde{\mathcal{W}}$. It is easy to check that $S_{Y}=\left[\theta_{e_{s}} \theta_{e_{r}}^{*} \mid 0<r<s, r, s \in \mathbb{Q}\right]$, where $\left(e_{q}\right)$ denotes the obvious basis in $l^{2}\left(\mathbb{Q}_{+}^{*}\right)$. Instead of $\mathbb{Q}$, we can as well take any countable subfield of $\mathbb{R}$.

Example 4.6. Up to now, we considered in fact only one example: the $a x+b$ group over an arbitrary l.c. ring with two natural subgroups. If $\mathcal{A}=\mathbb{R}$, this example is the easiest non-trivial example of a matched pair of real Lie groups (and the only non-trivial example when $G$ is of dimension 2). But, there are a lot of examples of matched pairs of algebraic groups, even in low dimensions, see e.g. [20]. Often, the field $\mathbb{R}$ or $\mathbb{C}$ can be replaced by any l.c. ring $\mathcal{A}$ with $\mathcal{A} \backslash \mathcal{A}^{\star}$ of measure zero and $\mathcal{A} \neq\{0\}$. Fix such a l.c. ring $\mathcal{A}$.

We give two examples. First, define $G=G L_{2}(\mathcal{A})$, the two by two matrices over $\mathcal{A}$. Take

$$
G_{1}=\left(\begin{array}{cc}
\mathcal{A}^{\star} & \mathcal{A} \\
0 & 1
\end{array}\right) \quad \text { and } \quad G_{2}=\left(\begin{array}{cc}
1 & 0 \\
\mathcal{A} & \mathcal{A}^{\star}
\end{array}\right) \text {. }
$$

Observe that $G_{1}$ and $G_{2}$ are conjugated by $\left(\begin{array}{ll}0 & 1 \\ 1 & 0\end{array}\right)$. 
Next, we give an example in the same spirit as [19], Section 5.4. Fix $q \in \mathcal{A}$ and suppose $q$ to be central. Define on $\mathcal{B}_{q}:=\mathcal{A}^{4}$ the structure of a l.c. ring by putting

$$
\left(\begin{array}{ll}
a & b \\
c & d
\end{array}\right)_{q}+\left(\begin{array}{ll}
a^{\prime} & b^{\prime} \\
c^{\prime} & d^{\prime}
\end{array}\right)_{q}=\left(\begin{array}{ll}
a+a^{\prime} & b+b^{\prime} \\
c+c^{\prime} & d+d^{\prime}
\end{array}\right)_{q} \quad \text { and } \quad\left(\begin{array}{ll}
a & b \\
c & d
\end{array}\right)_{q} \cdot\left(\begin{array}{ll}
a^{\prime} & b^{\prime} \\
c^{\prime} & d^{\prime}
\end{array}\right)_{q}=\left(\begin{array}{cc}
a a^{\prime}+q b c^{\prime} & a b^{\prime}+b d^{\prime} \\
c a^{\prime}+d c^{\prime} & d d^{\prime}+q c b^{\prime}
\end{array}\right)_{q} \text {. }
$$

Defining $\pi_{q}\left(\begin{array}{ll}a & b \\ c & d\end{array}\right)_{q}=\left(\begin{array}{ll}a & b \\ q c & d\end{array}\right) \oplus\left(\begin{array}{ll}a & q b \\ c & d\end{array}\right)$, we identify $\mathcal{B}_{q}$ with the closed subring of $M_{2}(\mathcal{A}) \oplus M_{2}(\mathcal{A})$ consisting of the elements $\left(\begin{array}{ll}a & b \\ c & d\end{array}\right) \oplus\left(\begin{array}{ll}a & b^{\prime} \\ c^{\prime} & d\end{array}\right)$ satisfying $b^{\prime}=q b$ and $c=q c^{\prime}$. If $q \in \mathcal{A}^{\star}$, the first component of $\pi_{q}$ gives an isomorphism $\mathcal{B}_{q} \cong M_{2}(\mathcal{A})$.

Define $G^{q}=\left(\mathcal{B}_{q}\right)^{\star}$ and

$$
G_{1}^{q}=\left\{\left(\begin{array}{ll}
a & b \\
0 & d
\end{array}\right)_{q} \mid a, d \in \mathcal{A}^{\star}, b \in \mathcal{A}\right\} \quad \text { and } \quad G_{2}^{q}=\left\{\left(\begin{array}{ll}
1 & 0 \\
x & 1
\end{array}\right)_{q} \mid x \in \mathcal{A}\right\} .
$$

Observe that $G_{1}^{q} \cong G_{1}^{1} \cong$ the group of upper triangular matrices in $G L_{2}(\mathcal{A})$ and $G_{2}^{q} \cong G_{2}^{1} \cong(\mathcal{A},+)$ for all $q \in \mathcal{A}$.

For any $q \in \mathcal{A}$, we get a bicrossed product l.c. quantum group $(M, \delta)_{q}$ whose right regular representation $W_{q}$ lives on the constant Hilbert space $L^{2}\left(G_{2}^{1} \times G_{1}^{1}\right)$. It is easy to check that the application $q \mapsto W_{q}$ is strongly* continuous. So, we have a continuous family of l.c. quantum groups. If $q \in \mathcal{A}^{\star}$, it is clear that $(M, \delta)_{q} \cong(M, \delta)_{1}$, because then, the first component of $\pi_{q}$ is an isomorphism. On the other hand, if $q=0$, we observe that $G_{2}^{0}$ is a normal subgroup of $G^{0}$. So, $G_{1}^{0}$ acts by automorphisms on $(\mathcal{A},+)$ and $G^{0} \cong G_{1}^{0} \ltimes \mathcal{A}$. So, $(M, \delta)_{0}$ is commutative and isomorphic to $\left(L^{\infty}(H), \delta_{H}\right)$, where $H:=G_{1}^{0} \ltimes \hat{\mathcal{A}}$ and $\hat{\mathcal{A}}$ is the Pontryagin dual of $(\mathcal{A},+)$.

In this precise sense, the l.c. quantum groups $(M, \delta)_{q}$ are continuous deformations of the l.c. group $H$.

If $\mathcal{A}$ is commutative, we can remain closer to [19], Section 5.4 and quotient out the center of $G^{q}$ consisting of the matrices $\left(\begin{array}{cc}a & 0 \\ 0 & a\end{array}\right)_{q}$, for $a \in \mathcal{A}^{\star}$.

\section{Concluding remarks}

\section{a) Pentagonal transformations}

Following [3], we call $v: X \times X \rightarrow X \times X$ a pentagonal transformation, when $X$ is a (standard) measure space and $v$ is a measure class isomorphism satisfying the pentagonal relation

$$
v_{23} \circ v_{13} \circ v_{12}=v_{12} \circ v_{23} .
$$

Associated to any pentagonal transformation $v$, we have a multiplicative unitary $V$ on the Hilbert space $L^{2}(X)$, defined by $(V \xi)(x, y)=d(x, y)^{1 / 2} \xi(v(x, y))$, where $d$ is the right Radon-Nikodym derivative.

Not all pentagonal transformations are nice: in Remark 4.5, we obtained a pentagonal transformation such that $S_{V}$ and $\hat{S}_{V}$ are not even $\mathrm{C}^{*}$-algebras.

In [3], there was given a natural sufficient condition for a pentagonal transformation to be good. As explained in the introduction, the result in [3] is incorrect as stated, but can be repaired as follows.

Proposition 5.1. Let $v$ be a pentagonal transformation on $X$. Introduce binary composition laws on $X$ by writing $v(x, y)=(x \bullet y, x \sharp y)$. Suppose that the transformations $\phi(x, y)=(x \bullet y, y)$ and $\eta(x, y)=(x, x \sharp y)$ are measure class isomorphisms.

Then, there exists a matched pair $G_{1}, G_{2}$ of closed subgroups of a l.c. group $G$ (in the sense of Definition 3.1), commuting actions on $X$ of $G_{2}$ on the left and $G_{1}$ on the right, and $a G_{2} \times G_{1}$-equivariant measurable map $f: X \rightarrow G$, such that

$$
v(x, y)=\left(x \cdot p_{1}\left(p_{2}(f(x))^{-1} f(y)\right), p_{2}(f(x))^{-1} \cdot y\right) \quad \text { for almost all } \quad(x, y) \in X \times X .
$$


We sketch the proof of this result, following [3] (there only appears an error in the proof of Proposition 3.4 a) of [3]). In order to find $G_{1}, G_{2}$ and $G$, we write $v^{-1}(x, y)=(x \diamond y, x * y)$ and further, $\psi^{\prime}(x, y)=(y * x, y)$, $w(a, b, c, d)=(a \bullet(b \sharp c), d *(b \bullet c), c, d)$. One can check that $\phi$ and $\psi^{\prime}$ are pentagonal transformations on $X$ and $w$ is a pentagonal transformation on $X \times X$. Because the pentagonal transformations $\phi, \psi^{\prime}, w$ are all of the form $(r, s) \mapsto(\ldots, s)$, it follows from Lemme 2.1 in [3] that we can find l.c. groups $G_{1}, G_{2}$ and $G$, right actions of $G_{i}$ on $X$ and of $G$ on $X \times X$, and equivariant measurable maps $f_{i}: X \rightarrow G_{i}, F: X \times X \rightarrow G$ such that

$$
\phi(x, y)=\left(x \cdot f_{1}(y), y\right), \quad \psi^{\prime}(x, y)=\left(x \cdot f_{2}(y), y\right) \quad \text { and } \quad w(a, b, c, d)=((a, b) \cdot F(c, d), c, d) .
$$

One also verifies that $w=\psi_{24}^{\prime} v_{21} \phi_{13} v_{21}^{-1}$.

Denote by $V$ the multiplicative unitary associated to $v$ and by $V^{1}, V^{2}$ and $W$ the multiplicative unitaries associated to $\phi, \psi^{\prime}$ and $w$, resp. So, we have $W=V_{21}^{*} V_{13}^{1} V_{21} V_{24}^{2}$. Hence, the group von Neumann algebras of $G_{1}$ and $G_{2}$ are von Neumann subalgebras of the group von Neumann algebra of $G$ and so, $G_{1}$ and $G_{2}$ can be considered as closed subgroups of $G$. Under this identification, one has $F(c, d)=f_{1}(c) f_{2}(d)$ almost everywhere. Because $F$ is surjective (from a measure theoretic point of view), it follows that the complement of $G_{1} G_{2}$ has measure zero. From the formula for $W$, we also get that $L^{\infty}(G) \subset L^{\infty}\left(G_{1} \times G_{2}\right)$ and we have to prove that we have an equality. Then, $G_{1} \cap G_{2}=\{e\}$ and we have a matched pair $G_{1}, G_{2}$ in $G$. Denote by $-\sigma$-weak the weak closure. Then,

$$
\begin{aligned}
L^{\infty}(G) & =\left[(\omega \otimes \iota \otimes \iota)\left(V_{21}^{*} V_{13}^{1} V_{21} V_{24}^{2}\right) \mid \omega \in \mathcal{L}\left(L^{2}(X \times X)\right)_{*}\right]^{-\sigma \text {-weak }} \\
& =\left[(\omega \otimes \iota \otimes \iota)\left(V_{13}^{1}\left(\left(L^{\infty}(X) \otimes 1\right) V\left(1 \otimes L^{\infty}(X)\right)\right)_{21} V_{24}^{2}\right) \mid \omega \in \mathcal{L}\left(L^{2}(X \times X)\right)_{*}\right]^{-\sigma \text {-weak }} .
\end{aligned}
$$

If $H, K \in L^{\infty}(X)$, we observe that $(H \otimes 1) V(1 \otimes K) V^{*}$ is the function $(x, y) \mapsto H(x) K(x \sharp y)$. As the transformation $(x, y) \mapsto(x, x \sharp y)$ is a measure class isomorphism, the weak closure of these functions is the whole of $L^{\infty}(X \times X)$. So,

$$
\begin{aligned}
L^{\infty}(G) & =\left[(\omega \otimes \iota \otimes \iota)\left(V_{13}^{1} L^{\infty}(X)_{1} V_{21} V_{24}^{2}\right) \mid \omega \in \mathcal{L}\left(L^{2}(X \times X)\right)_{*}\right]^{-\sigma \text {-weak }} \\
& =\left[(\omega \otimes \iota \otimes \iota)\left(V_{13}^{1}\left(\hat{S}_{V^{1}}^{\prime} L^{\infty}(X)\right)_{1} V_{21} V_{24}^{2}\right) \mid \omega \in \mathcal{L}\left(L^{2}(X \times X)\right)_{*}\right]^{-\sigma \text {-weak }} \\
& =\left[\left(L^{\infty}\left(G_{1}\right) \otimes 1\right)(\omega \otimes \iota \otimes \iota)\left(V_{24}^{2}\right) \mid \omega \in \mathcal{L}\left(L^{2}(X \times X)\right)_{*}\right]^{-\sigma \text {-weak }}=L^{\infty}\left(G_{1} \times G_{2}\right),
\end{aligned}
$$

where we used that $\hat{S}_{V^{1}}^{\prime} L^{\infty}(X)$ is weakly dense in $\mathcal{L}\left(L^{2}(X)\right)$. We conclude that we indeed get a matched pair $G_{1}, G_{2}$ in $G$.

Because $\eta^{\prime}(x, y)=(y \sharp x, y)$ is a measure class isomorphism, we associate with it a unitary operator $B$ on $L^{2}(X \times X)$. One checks that $\psi_{23}^{\prime} \eta_{12}^{\prime} \eta_{13}^{\prime}=\eta_{12}^{\prime} \psi_{23}^{\prime}$ and hence, $B_{13} B_{12} V_{23}^{2}=V_{23}^{2} B_{12}$. It follows that $B^{*}$ is a representation of $V^{2}$. Hence, $B \in \mathcal{L}\left(L^{2}(X)\right) \otimes L^{\infty}\left(G_{2}\right)$. It follows that there exists a left action $\triangleright$ of $G_{2}$ on $X$ such that $y \sharp x=f_{2}(y) \triangleright x$ almost everywhere.

Analogously, we can find a right action of $G_{1}$ on $X$ such that $x \triangleleft f_{1}(y)^{-1}=x \diamond y$. The left action of $G_{2}$ and the right action of $G_{1}$ commute and defining $f(x)=f_{1}(x) f_{2}(x)^{-1}$, we have a $G_{2} \times G_{1}$-equivariant measurable map $f: X \rightarrow G$. One can show that this map and the commuting actions of $G_{1}$ and $G_{2}$ satisfy the conclusion of the proposition.

Remark 5.2. Combining Proposition 5.1 and Proposition 3.8, we observe that the multiplicative unitary $V$ associated to a good pentagonal transformation $v$ is precisely a covariant image of the regular representation of the associated bicrossed product. In particular, $\left(S_{V}, \delta_{V}\right)$ is always a bicrossed product l.c. quantum group.

Remark 5.3. In the beginning of Section 3, we started with a matched pair $G_{1}, G_{2}$ of closed subgroups of $G$ and constructed with them a ${ }^{*}$-automorphism $\tau: L^{\infty}\left(G_{1} \times G_{2}\right) \rightarrow L^{\infty}\left(G_{2} \times G_{1}\right)$ such that $\tau \sigma$ is a matching of $\left(L^{\infty}\left(G_{2}\right), \delta_{2}\right)$ and $\left(L^{\infty}\left(G_{1}\right), \delta_{1}^{\text {op }}\right)$ with trivial cocycles, in the sense of [19], Definition 2.1. Suppose now that, conversely, $\tau$ is such that $\tau \sigma$ is a matching. Hence, $\tau$ is a faithful ${ }^{*}$-homomorphism satisfying

$$
(\tau \otimes \iota)(\iota \otimes \tau)\left(\delta_{1} \otimes \iota\right)=\left(\iota \otimes \delta_{1}\right) \tau \quad \text { and } \quad(\iota \otimes \tau)(\tau \otimes \iota)\left(\iota \otimes \delta_{2}\right)=\left(\delta_{2} \otimes \iota\right) \tau .
$$

Defining $\alpha$ and $\beta$ to be the restrictions of $\tau$ to $L^{\infty}\left(G_{1}\right)$ and $L^{\infty}\left(G_{2}\right)$ respectively, we get a left action of $G_{2}$ on $L^{\infty}\left(G_{1}\right)$ and a right action of $G_{1}$ on $L^{\infty}\left(G_{2}\right)$. Hence, we can write $\tau(F)(s, g)=F\left(\alpha_{s}(g), \beta_{g}(s)\right)$ for 
almost all $g, s$ and where $\left(\alpha_{s}\right)$ and $\left(\beta_{g}\right)$ are actions of $G_{2}$, resp. $G_{1}$ by measure class isomorphisms of $G_{1}$, resp. $G_{2}$. We can define with the same formula as in Definition 3.3, the multiplicative unitary $W$, which corresponds to the pentagonal transformation

$$
v(s, g, t, h)=\left(s, g \alpha_{\beta_{g}(s)^{-1} t}(h), \beta_{g}(s)^{-1} t, h\right)
$$

on $X:=G_{2} \times G_{1}$. Corresponding to $v$, we have the mappings $\phi$ and $\eta$ as above. It is clear that $\eta$ is a measure class isomorphism. We prove that the same holds for $\phi$. Because we have a pentagonal transformation, we can define the (measure theoretically) surjective mapping $P(x, y)=x \bullet y$. Defining the measure class isomorphism $u_{\alpha}(s, g)=\left(s, \alpha_{s}(g)\right)$ and the surjective mapping $P_{1}(s, g, t, h)=(s, g h)$, one checks that $u_{\alpha} P=P_{1}\left(u_{\alpha} \times u_{\alpha}\right)$, where we use the relation $\alpha_{s}(g h)=\alpha_{s}(g) \alpha_{\beta_{g}(s)}(h)$ for almost all $s, g, h$, which follows from $\left(\iota \otimes \delta_{1}\right) \alpha=(\tau \otimes \iota)(\iota \otimes \alpha) \delta_{1}$. It follows that $P=u_{\alpha}^{-1} P_{1}\left(u_{\alpha} \times u_{\alpha}\right)$ and so $\phi=\left(u_{\alpha}^{-1} \times u_{\alpha}^{-1}\right) \phi_{24}^{1}\left(u_{\alpha} \times u_{\alpha}\right)$, where $\phi^{1}(g, h)=(g h, h)$. Hence, also $\phi$ is a measure class isomorphism and we can apply Proposition 5.1. We find a l.c. group $G$ and going through the construction, we observe that we identify $G_{1}$ and $G_{2}$ with closed subgroups of $G$ such that $G_{1}, G_{2}$ is a matched pair and $\alpha_{s}(g)=p_{1}(s g), \beta_{g}(s)=p_{2}(s g)$.

\section{b) Regularity and semi-regularity}

We will give several characterizations of regularity and semi-regularity of l.c. quantum groups. In fact, it will become clear that it is very amazing that there indeed exist non-semi-regular l.c. quantum groups.

Terminology 5.4. A l.c. quantum group is said to be regular, resp. semi-regular, if its right (or, equivalently, left) regular representation is a regular, resp. semi-regular multiplicative unitary.

Let $V$ be the right regular representation of a fixed l.c. quantum group $(M, \delta)$, as in Section 2. Denote by $S$ its underlying $\mathrm{C}^{*}$-algebra as in Equation (2.1).

In the proof of Proposition 2.6, we introduced the modular conjugations $J$ and $\hat{J}$, that we will use extensively. They are anti-unitary operators on the Hilbert space $H$, which is the GNS-space of the invariant weights. Observe that $J M J=M^{\prime}, \hat{J} \hat{M} \hat{J}=\hat{M}^{\prime}, \hat{J} S \hat{J}=S$ and $J \hat{S} J=\hat{S}$.

When we write $\mathcal{K}$, we always mean $\mathcal{K}(H)$.

From Proposition 2.6, we know that regularity is equivalent with $\mathcal{K}=[S \hat{S}]$ and this is not always the case. However, if we put slightly more, we do get $\mathcal{K}$.

Lemma 5.5. We have $[$ JSJ $S \hat{S}]=\mathcal{K}$.

Proof. From Equation (2.2) and using the notation $U=J \hat{J}$, we know that, up to a scalar

$$
(1 \otimes U) \Sigma=(U \otimes U) V^{*}(1 \otimes U) \Sigma V^{*} \Sigma(U \otimes 1) V^{*} .
$$

Applying $\omega \otimes \iota$, it follows that

$$
\mathcal{K}=\left[(\omega \otimes \iota)\left((U \otimes U) V^{*}(1 \otimes U) \Sigma V^{*} \Sigma(U \otimes 1) V^{*}\right) \mid \omega \in \mathcal{L}(H)_{*}\right] .
$$

Because $\mathcal{K}=[J S J \mathcal{K} S]$ and because $V \in \mathrm{M}(\mathcal{K} \otimes S)$, it follows that

$$
\mathcal{K}=\left[J S J(\omega \otimes \iota)\left(\Sigma V^{*} \Sigma\right) S \mid \omega \in \mathcal{L}(H)_{*}\right]=[J S J \hat{S} S] .
$$

Because $[\hat{S} S]$ is a $\mathrm{C}^{*}$-algebra, we are done.

Recall that $V \in \mathrm{M}(\mathcal{K} \otimes S)$ and $S=\left[(\omega \otimes \iota)(V) \mid \omega \in \mathcal{L}(H)_{*}\right]$. So, the following result is quite surprising.

Proposition 5.6. The l.c. quantum group $(M, \delta)$ is semi-regular if and only if

$$
\left[(\omega \otimes \iota)\left(V^{*}(1 \otimes S) V\right) \mid \omega \in \mathcal{L}(H)_{*}\right] \cap S \neq\{0\} .
$$

If this is the case, we have $S \subset\left[(\omega \otimes \iota)\left(V^{*}(1 \otimes S) V\right) \mid \omega \in \mathcal{L}(H)_{*}\right]$. The quantum group is regular if and only if the equality holds. 
Proof. We first claim that in general

$$
\left[\hat{S} J S J(\omega \otimes \iota)\left(V^{*}(1 \otimes S) V\right) \mid \omega \in \mathcal{L}(H)_{*}\right]=[\hat{S} J S J] .
$$

We make the following calculation, where we use the pentagonal equation, Lemma 5.5 and the formula $V^{*}=(J \otimes \hat{J}) V(J \otimes \hat{J})$.

$$
\begin{aligned}
{\left[\hat{S} J S J(\omega \otimes \iota)\left(V^{*}(1 \otimes S) V\right) \mid \omega \in \mathcal{L}(H)_{*}\right] } & =\left[(\omega \otimes \iota \otimes \mu)\left(V_{23} V_{12}^{*}(1 \otimes J S J S \otimes 1) V_{12}\right) \mid \omega, \mu \in \mathcal{L}(H)_{*}\right] \\
& =\left[(\omega \otimes \iota \otimes \mu)\left(V_{13}^{*} V_{12}^{*} V_{23}(1 \otimes J S J \otimes 1) V_{12}\right) \mid \omega, \mu \in \mathcal{L}(H)_{*}\right] \\
& =\left[(\omega \otimes \iota)\left(V^{*}(1 \otimes \hat{S} J S J S) V\right) \mid \omega \in \mathcal{L}(H)_{*}\right] \\
& =\hat{J}\left[(\iota \otimes \omega)\left(\Sigma V(1 \otimes \mathcal{K}) V^{*} \Sigma\right) \mid \omega \in \mathcal{L}(H)_{*}\right] \hat{J} \\
& =\hat{J}\left[\mathcal{C}(V) \mathcal{C}(V)^{*}\right] \hat{J}=[\hat{S} J S J],
\end{aligned}
$$

where, in the end, we used the calculation of $\mathcal{C}(V)$ from the proof of Proposition 2.6. This proves our claim. Suppose that $a \neq 0$ and $a \in\left[(\omega \otimes \iota)\left(V^{*}(1 \otimes S) V\right) \mid \omega \in \mathcal{L}(H)_{*}\right] \cap S$. Take $b \in S$ and $x \in \hat{S}$ such that $x J b J a \neq 0$. Combining our claim with Lemma 5.5, we see that $x J b J a \in[\hat{S} J S J] \cap \mathcal{K}$. Hence, $[\hat{S} J S J] \cap \mathcal{K} \neq\{0\}$. Because the $\mathrm{C}^{*}$-algebra $[\hat{S} J S J]$ acts irreducibly on $H$, it follows that $\mathcal{K} \subset[\hat{S} J S J]$ and so, the l.c. quantum group is semi-regular.

Conversely, suppose that the l.c. quantum group is semi-regular. Then,

$$
\begin{aligned}
{\left[(\omega \otimes \iota)\left(V^{*}(1 \otimes S) V\right) \mid \omega \in \mathcal{L}(H)_{*}\right] } & =\left[(\mu \otimes \omega \otimes \iota)\left(V_{23}^{*} V_{13}^{*} V_{23}\right) \mid \omega, \mu \in \mathcal{L}(H)_{*}\right] \\
& =\left[(\mu \otimes \omega \otimes \iota)\left(V_{23}^{*} V_{12} V_{23}\right) \mid \omega, \mu \in \mathcal{L}(H)_{*}\right] \\
& =\left[(\omega \otimes \iota)\left(V^{*}(S \otimes 1) V\right) \mid \omega \in \mathcal{L}(H)_{*}\right] \\
& =\left[(\omega \otimes \iota)\left(V^{*}(\hat{J} \hat{S} \hat{J} S \otimes 1) V\right) \mid \omega \in \mathcal{L}(H)_{*}\right] \\
& \supset\left[(\omega \otimes \iota)\left(V^{*}(\mathcal{K} \otimes 1) V\right) \mid \omega \in \mathcal{L}(H)_{*}\right]=S
\end{aligned}
$$

If the l.c. quantum group is regular, the $\supset$ above is an equality. If, finally, $\left[(\omega \otimes \iota)\left(V^{*}(1 \otimes S) V\right) \mid \omega \in\right.$ $\left.\mathcal{L}(H)_{*}\right]=S$, it follows from our claim above and Lemma 5.5 that

$$
[\hat{S} J S J]=[\hat{S} J S J S]=\mathcal{K} .
$$

This precisely gives the regularity.

Another characterization of semi-regularity is in terms of coactions of l.c. quantum groups.

Let $B$ be a $\mathrm{C}^{*}$-algebra and $\alpha: B \rightarrow \mathrm{M}(S \otimes B)$ a non-degenerate *-homomorphism satisfying $(\iota \otimes \alpha) \alpha=(\delta \otimes \iota) \alpha$. We say that $\alpha$ is a coaction of $S$ on $B$.

A seemingly natural definition for the continuous elements under the coaction is

$$
T:=\left[(\omega \otimes \iota) \alpha(B) \mid \omega \in \mathcal{L}(H)_{*}\right] .
$$

Proposition 5.7. The l.c. quantum group $(M, \delta)$ is semi-regular if and only if the above closed linear space $T$ of continuous elements is a $C^{*}$-algebra for all coactions $\alpha$ of $S$ on a $C^{*}$-algebra $B$.

Proof. Suppose first that $(M, \delta)$ is semi-regular. Then,

$$
\begin{aligned}
T & \supset\left[(\omega \otimes \iota) \alpha(y(\mu \otimes \iota) \alpha(x)) \mid \omega, \mu \in \mathcal{L}(H)_{*}, x, y \in B\right] \\
& =\left[(\mu \otimes \omega \otimes \iota)\left(\alpha(y)_{23} V_{12} \alpha(x)_{13} V_{12}^{*}\right) \mid \omega, \mu \in \mathcal{L}(H)_{*}, x, y \in B\right] \\
& =\left[(\mu \otimes \omega \otimes \iota)\left(\alpha(y)_{23}((\mathcal{K} \otimes 1) V(1 \otimes \mathcal{K}))_{12} \alpha(x)_{13}\right) \mid \omega, \mu \in \mathcal{L}(H)_{*}, x, y \in B\right] \\
& \supset\left[(\mu \otimes \omega \otimes \iota)\left(\alpha(y)_{23}(\mathcal{K} \otimes \mathcal{K} \otimes 1) \alpha(x)_{13}\right) \mid \omega, \mu \in \mathcal{L}(H)_{*}, x, y \in B\right]=[T T] .
\end{aligned}
$$

Hence, $T$ is an algebra. It is clear that $T^{*}=T$ and so, $T$ is a $\mathrm{C}^{*}$-algebra. 
If conversely, the space of continuous elements is always a $\mathrm{C}^{*}$-algebra, we define $B:=\mathcal{K}(H \oplus \mathbb{C})$. Consider the left regular representation $W \in \mathrm{M}(S \otimes \mathcal{K})$ satisfying $(\delta \otimes \iota)(W)=W_{13} W_{23}$ and put $X:=W \oplus 1 \in \mathrm{M}(S \otimes B)$. Define $\alpha(x)=X^{*}(1 \otimes x) X$ for all $x \in B$. Writing $\theta_{\xi}$ for the obvious operator in $\mathcal{K}(\mathbb{C}, H)$ whenever $\xi \in H$, we observe that

$$
T=\left\{\left(\begin{array}{cc}
{\left[(\omega \otimes \iota)\left(W^{*}(1 \otimes \mathcal{K}) W\right) \mid \omega \in \mathcal{L}(H)_{*}\right]} & \theta_{\xi} \\
\theta_{\eta}^{*} & \mathbb{C}
\end{array}\right) \mid \xi, \eta \in H\right\} .
$$

So, because $T$ is a $\mathrm{C}^{*}$-algebra, we get

$$
\mathcal{K} \subset\left[(\omega \otimes \iota)\left(W^{*}(1 \otimes \mathcal{K}) W\right) \mid \omega \in \mathcal{L}(H)_{*}\right]
$$

Using the fact that $W=\Sigma(U \otimes 1) V\left(U^{*} \otimes 1\right) \Sigma$, we conclude that

$$
\mathcal{K} \subset U\left[(\iota \otimes \omega)\left(V^{*} \Sigma(1 \otimes \mathcal{K}) \Sigma V\right) \mid \omega \in \mathcal{L}(H)_{*}\right] U^{*}=U\left[\mathcal{C}(V)^{*} \mathcal{C}(V)\right] U^{*}=[S \hat{S}]
$$

So, $(M, \delta)$ is semi-regular.

We conclude that the notion of continuous elements is problematic for coactions of non-semi-regular quantum groups. Hence, it is not surprising that is equally problematic to define continuous coactions.

There are at least two natural definitions. Let $\alpha: B \rightarrow \mathrm{M}(S \otimes B)$ be a coaction.

- We call $\alpha$ continuous in the weak sense when $B=\left[(\omega \otimes \iota) \alpha(B) \mid \omega \in \mathcal{L}(H)_{*}\right]$.

- We call $\alpha$ continuous in the strong sense when $S \otimes B=[\alpha(B)(S \otimes 1)]$.

We have the following result.

Proposition 5.8. Consider a coaction $\alpha: B \rightarrow \mathrm{M}(S \otimes B)$ of $S$ on $B$. If $(M, \delta)$ is regular, continuity in the weak sense implies continuity in the strong sense.

If $(M, \delta)$ is semi-regular, but not regular, there exists a coaction $\alpha$ that is continuous in the weak sense, but not in the strong sense.

Proof. If $(M, \delta)$ is regular, we know from [2] that $[(\mathcal{K} \otimes 1) V(1 \otimes S)]=\mathcal{K} \otimes S$. So, if $\alpha$ is continuous in the weak sense, we get

$$
\begin{aligned}
{[\alpha(B)(S \otimes 1)] } & =\left[\alpha((\omega \otimes \iota) \alpha(B))(S \otimes 1) \mid \omega \in \mathcal{L}(H)_{*}\right] \\
& =\left[(\omega \otimes \iota \otimes \iota)\left(V_{12} \alpha(B)_{13} V_{12}^{*}(\mathcal{K} \otimes S \otimes 1)\right) \mid \omega \in \mathcal{L}(H)_{*}\right] \\
& =\left[(\omega \otimes \iota \otimes \iota)\left(V_{12} \alpha(B)_{13}(1 \otimes S \otimes 1)\right) \mid \omega \in \mathcal{L}(H)_{*}\right] \\
& =\left[(\omega \otimes \iota \otimes \iota)\left(((\mathcal{K} \otimes 1) V(1 \otimes S))_{12} \alpha(B)_{13}\right) \mid \omega \in \mathcal{L}(H)_{*}\right] \\
& =S \otimes\left[(\omega \otimes \iota) \alpha(B) \mid \omega \in \mathcal{L}(H)_{*}\right]=S \otimes B .
\end{aligned}
$$

Hence, $\alpha$ is continuous in the strong sense.

Suppose now that $(M, \delta)$ is semi-regular, but not regular. Define

$$
B:=\left\{\left(\begin{array}{cc}
{[\hat{S} S]} & \theta_{\xi} \\
\theta_{\eta}^{*} & \mathbb{C}
\end{array}\right) \mid \eta, \xi \in H\right\} \quad \text { with } \quad \alpha\left(\begin{array}{cc}
x & \theta_{\xi} \\
\theta_{\eta}^{*} & \lambda
\end{array}\right)=\left(\begin{array}{cc}
W^{*}(1 \otimes x) W & W^{*}\left(1 \otimes \theta_{\xi}\right) \\
\left(1 \otimes \theta_{\eta}^{*}\right) W & 1 \otimes \lambda
\end{array}\right)
$$

where $W \in \mathrm{M}(S \otimes \hat{J} \hat{S} \hat{J})$ is again the left regular representation. It is easy to verify that $\alpha$ is a coaction which is continuous in the weak sense, but not in the strong sense. 


\section{References}

[1] S. BAAJ, Représentation régulière du groupe quantique des déplacements de Woronowicz. Astérisque 232 (1995), 11-48.

[2] S. BaAj \& G. Skandalis, Unitaires multiplicatifs et dualité pour les produits croisés de $\mathrm{C}^{*}$-algèbres. Ann. Scient. Ec. Norm. Sup., 4 e série, 26 (1993), 425-488.

[3] S. Baaj \& G. Skandalis, Transformations pentagonales. C.R. Acad. Sci., Paris, Sér. I 327 (1998), 623-628.

[4] S. BaAj \& S. Vaes, Double crossed products of locally compact quantum groups. J. Inst. Math. Jussieu, to appear.

[5] J.-B. Bost \& A. Connes, Hecke algebras, type III factors and phase transitions with spontaneous symmetry breaking in number theory. Selecta Math. (N.S.) 1 (1995), 411-457.

[6] P. Desmedt, J. Quaegebeur \& S. Vaes, Amenability and the bicrossed product construction. Ill. J. Math., to appear.

[7] M. Enock \& J-M. Schwartz, Kac Algebras and Duality of Locally Compact Groups, SpringerVerlag, 1992.

[8] T. FACK, Quelques remarques sur le spectre des $\mathrm{C}^{*}$-algèbres de feuilletages. Bull. Soc. Math. Belg. Sér. B 36 (1984), 113-129.

[9] G.I. Kac, Ring groups and the principle of duality, I, II. Trans. Moscow Math. Soc. (1963), 291-339, (1965), 94-126.

[10] G.I. KAC, Extensions of groups to ring groups. Math. USSR Sbornik 5 (1968), 451-474.

[11] G.I. KaC \& L.I. Vainerman, Nonunimodular ring groups and Hopf-von Neumann algebras. Math. USSR. Sbornik 23 (1974), 185-214.

[12] R.M. Kashaev \& S.M. Sergeev, On pentagon, ten-term, and tetrahedron relations. Comm. Math. Phys. 195 (1998), 309-319.

[13] J. Kustermans, Locally compact quantum groups in the universal setting. Internat. J. Math. 12 (2001), 289-338.

[14] J. Kustermans \& S. Vaes, Locally compact quantum groups. Ann. Scient. Ec. Norm. Sup., $4^{e}$ série 33 (2000), 837-934.

[15] J. Kustermans \& S. VAes, Locally compact quantum groups in the von Neumann algebraic setting. Math. Scand. 92 (1) (2003), 68-92.

[16] S. MAJID, Hopf-von Neumann algebra bicrossproducts, Kac algebra bicrossproducts, and the classical Yang-Baxter equations. J. Funct. Anal. 95 (1991), 291-319.

[17] M. A. RiEffel, Strong Morita equivalence of certain transformation group $\mathrm{C}^{*}$-algebras. Math. Ann., 222 (1976), 71-97.

[18] M. TAKeuchi, Matched pairs of groups and bismash products of Hopf algebras. Comm. Algebra 9 (1981), 841-882.

[19] S. Vaes \& L. Vainerman, Extensions of locally compact quantum groups and the bicrossed product construction. Adv. in Math. 175 (1) (2003), 1-101.

[20] S. Vaes \& L. Vainerman, On low-dimensional locally compact quantum groups. In Locally Compact Quantum Groups and Groupoids. Proceedings of the Meeting of Theoretical Physicists and Mathematicians, Strasbourg, February 21 - 23, 2002., Ed. L. Vainerman, IRMA Lectures on Mathematics and Mathematical Physics, Walter de Gruyter, Berlin, New York (2003), pp. 127-187. 
[21] S.L. Woronowicz, From multiplicative unitaries to quantum groups. Int. J. Math. 7 (1) (1996), $127-149$.

[22] S.L. Woronowicz, Twisted $S U(2)$ group. An example of a non-commutative differential calculus. Publ. RIMS, Kyoto University 23 (1987), 117-181. 